\title{
Feld der Technik
}

Die Personengruppe der Techniker und Ingenieure positionierte sich einerseits im übergeordneten sozialen Raum relational zu konkurrierenden Professionen wie Juristen und Ökonomen, die im Wettbewerb um gut entlohnte Stellen in der Privatwirtschaft, aber auch der öffentlichen Verwaltung standen, sowie spezifisch im Feld der Technik. ${ }^{1}$ Gerade der letzte Bezugsrahmen ermöglicht es, die Stellung und Beziehung der technischen Berufe und Bildungsgrade miteinander ins Verhältnis zu setzen. Das Feld der Technik wird zusätzlich um den kolonialen Bereich im Sinne eines Subfeldes erweitert. Jener Bereich lässt sich auch für andere Felder denken und ist daher nicht auf das der Technik beschränkt. Von einem eigenständigen Feld der Kolonialtechnik ist jedoch nicht auszugehen, da die entsprechenden Charakteristika nicht vorliegen. ${ }^{2}$

Die Begrifflichkeit des Feldes der Technik ist in der historischen Forschung nicht neu. Seine Verwendung ist in den meisten Fällen mit den Arbeiten Pierre Bourdieus verknüpft, ohne dass damit jedoch eine intensive Auseinandersetzung mit dessen Konzeption hinsichtlich der Spezifik eines Feldes der Technik einhergeht. Häufig bleibt es beim benannten Label, das lediglich als Hintergrundfolie für die Präsentation der eigentlichen historischen Forschung dient. Bisherige Studien lassen daher eine Auseinandersetzung mit dem Feld selbst, vor allem aber mit seiner Genese vermissen. Sie betonen dafür selektiv andere Aspekte aus Bourdieus theoretischem Konzept wie das Verhältnis von symbolischer Macht und Gewalt im Spannungsfeld von Geschlecht und Technik. ${ }^{3}$

Bei ihren Forschungen zu Ingenieurinnen in der Deutschen Demokratischen Republik verweist Karin Zachmann auf das Feld der Technik, das sich als Teil des sozialen Feldes konstruiert und sich durch die „spezifische Rationalität des technischen Handelns" seiner Akteure auszeichnet. Diese verorten sich in Relation zueinander, abhängig von ihrer Berufs- und Bildungsbiografie. ${ }^{4}$ Auch der Ingenieur und literarisch umtriebige Vertreter der deutschen Technokratiebewegung, Heinrich Hardensett, führte bereits in

1 Vgl. Weihe, Kultur und Technik, 1935, S. 99; Kocka, Kultur und Technik, 2012 [Zugriff: 12.6.2019].

2 Vgl. Bourdieu, Soziologische Fragen, 1993, S. 107.

3 Vgl. dazu beispielsweise Schreyer, Akademikerinnen, 20o8; Zachmann, Mobilisierung der Frauen, 2004. Allg. zur Etablierung und Dominanz einer als männlich empfundenen Technik: Paulitz, Mann und Maschine, 2012.

4 Zachmann, Mobilisierung der Frauen, 2004, S. 32. 
den 1920er-Jahren den Begriff der „technischen Rationalität“ im Munde, mit dem er die „Zweckmäßigkeit aller Maßnahmen“ umschreibt, die der Techniker oder Ingenieur dazu einsetzt, ein definiertes Ziel zu erreichen. ${ }^{5}$ Zum Verständnis dieser spezifischen "technischen Rationalität" bedarf es jedoch der Untersuchung der Genese des technischen Feldes. ${ }^{6}$

Fragen nach der sozialen Herkunft der technischen Experten wie auch nach den möglichen Gründen ihrer Berufswahl wird vor allem hinsichtlich einer Kategorisierung der Untergruppe der Kolonialingenieure nachgegangen. Im Gegensatz zu einer vollständigen kritischen Auseinandersetzung mit der verfügbaren umfangreichen Literatur zu diesem Themenbereich soll diese auf ein Maß begrenzt werden, wie es für die spätere, darauf aufbauende Argumentation notwendig ist. Im Hinblick auf die in vielen Bereichen bereits sehr dicht beforschte und beschriebene technische Berufsgruppe werden gezielt solche Diskurse und Entwicklungen herausgegriffen, die als essenziell für die weitere Thesenbildung gelten. Dementsprechend ist die eigentliche Genese des Ingenieurs sowohl als Begriff als auch als Profession im späten Mittelalter und der Renaissance nicht Teil der Analyse. ${ }^{7}$ Vielmehr liegt mein Hauptaugenmerk auf den Herausforderungen, Problemen und den sich daran anschließenden Entwicklungen, die bei sich zunehmend industrialisierenden europäischen Staaten während des 19. Jahrhunderts auftraten.

Ich werde untersuchen, wie diese junge Berufsgruppe darum stritt, gleichberechtigt ihren Platz neben etablierten gesellschaftlichen Gruppen einzunehmen. Die vermeintliche oder auch tatsächliche fehlende Anerkennung vonseiten der humanistischen Eliten, aber auch der Gesellschaft selbst animierte einige Vertreter der technischen Profession, nicht nur die Bedeutung der eigenen Berufsgruppe zu betonen, sondern auch für deren sozialen Aufstieg einzutreten. ${ }^{8}$ Eindeutige Konfrontationen blieben im Rahmen dieser Emanzipationsbewegung jedoch die Ausnahme. ${ }^{9}$ Vielmehr folgten die

5 Hardensett, Der Technische Mensch, 1932, S. 98. Synonym ließe sich mit Zillgen sogar vom „technisierten Geist“ sprechen: Zillgen, Staatspolitik, 1925, S. 3.

6 Vgl. Bourdieu, Regeln der Kunst, 1999.

7 Popplow, Unsichere Karrieren, 2006; Bayerl, Technische Intelligenz, 1978. Vgl. zum Begriff des Ingenieurs in der modernen Ausprägung: Kocka, Die Angestellten, 1981, S. 95-97.

8 Vgl. u. a. Münzinger, Baumeister einer besseren Welt, 1947, S. 220-227; Manegold, Emanzipation der Technik, 1977, S. 42; Lundgreen, Bild des Ingenieurs, 1994, S. 18; u. a. Herf, Reactionary Modernism, 1993, S. 152; beispielhaft Anonymus, Wirtschaftsingenieur, 1926.

9 Anonymus, Regierungsbaumeisterprüfung, 1945. Hierbei tat sich jedoch besonders jene Gruppe von Ingenieuren hervor, die sich in der deutschen Technokratiebewegung engagierte und ein klares Feindbild besaß: „Der stärkste Gegenspieler des Technikers ist heut der Politiker“: K. B., Technik und Politik, 1927, S. 512 f. Vgl. allg. zur deutschen Technokratiebewegung: Willeke, Technokratiebewegung, 1995. 
Ingenieure dem Credo, ihr Ziel mithilfe propagierter selbstloser Pflichterfüllung zu erreichen..$^{10}$ Es erscheint durchaus legitim, an dieser Stelle von einem spezifischen Habitus zu sprechen. Dieser stimmte mit der Doxa überein, dass Technik als solche neutral sei und die Aufgabe habe, der gesamten Menschheit zu dienen. ${ }^{11}$ Gesellschaftliche Probleme wurden als technische Hindernisse wahrgenommen, für die es einer bestimmten, objektiv ermittelbaren Lösung bedurfte. ${ }^{12}$ Diese Argumentation machten sich Ingenieure zu eigen, wenn sie diese auf die Begrifflichkeit der technischen Rationalität reduzierten und damit ein bestimmtes Weltbild für sich in Anspruch nahmen.

In einem ersten Schritt ist es wichtig, die Grenzen des Feldes der Technik zu definieren. Erscheint es aus heutiger Sicht unproblematisch, von einer klar abgegrenzten Gruppe der Ingenieure und Techniker zu sprechen, so verweist der englische Historiker und ausgewiesene Spezialist für die Geschichte des deutschen Ingenieurwesens, Kees Gispen, auf die Heterogenität jener Profession für das ausgehende 19. Jahrhundert. Hinsichtlich ihrer Ausbildung und sozialen Herkunft fand sich hier ein gemischter Personenkreis zusammen. ${ }^{13}$ Demzufolge verorte ich die Genese des Feldes der Technik historisch in jener Übergangszeit des 19. Jahrhunderts, in der die technische Ausbildung zunehmend institutionalisiert und vereinheitlicht wurde. Sowohl die Gruppe selbst als auch ihre Tätigkeitsfelder, in denen sie eingesetzt wurde, können als äußerst divers beschrieben werden und untergliederten sich in zahlreiche Fachbereiche mit differenzierten technischen Spezialisierungen.

Gispen leitet hieraus sogar die Frage ab, ob es sinnvoll erscheint oder überhaupt möglich ist, von den Ingenieuren ${ }^{14} \mathrm{zu}$ sprechen, da selbst die

10 Vgl. hierzu vor allem die Veröffentlichungen im Rahmen des Teilprojekts M im SFB 804 (2009 bis 2014) zum Fortschrittsversprechen von Technik und der Altruismusbehauptung der Ingenieure in der technokratischen Hochmoderne (1880-1970). Hänseroth spricht in diesem Zusammenhang von den "habitualisierten Altruismussuppositionen“, die einem kritischen Hinterfragen entzogen waren: Hänseroth, Technischer Fortschritt, 2013, S. 28o.

11 Noch 1959 ließ der Diplom-Ingenieur Karl Krüger verlauten: „Doch niemals wird die Technik unmoralisch sein, sondern nur der Mensch“: Krüger, Technik für alle Länder, 1959, S. 9.

12 Vgl. Dessauer, Philosophie der Technik, 1928, S. 19. Todt formuliert es prosaischer, wenn er den Ingenieur als „[k]ühn in seinen Plänen, selbstlos in der Hingabe seiner Arbeit an die Gesamtheit“ beschreibt: Anonymus, Reichsminister Dr. Todt, 1941; vgl. auch Hardensett, Der Technische Mensch, 1932, S. 124.

13 Vgl. Jarausch, Unfree Profession, 1990, S. 17 f.

14 Erst 1970/71 wurden nach einem erfolglosen bundespolitischen Anlauf Gesetze auf Landesebene erlassen, die das Tragen des Ingenieur-Titels regelten. Voraussetzung war ein erfolgreiches Studium an einer wissenschaftlichen Hochschule in einem überwiegend naturwissenschaftlichen oder technischen Studiengang: Kaiser, Ingenieure in der Bundesrepublik, 2006, S. 237. 
Bezeichnung Ingenieur für den zivilen Bereich erst in der zweiten Hälfte des 19. Jahrhunderts gebräuchlich wurde. ${ }^{15}$ Da sich in Deutschland ein staatlich garantiertes Ausbildungs- und Zugangswesen erst in jenem Jahrhundert entwickelte, wurden auch andere Kriterien diskutiert, die maßgeblich für die Berufsbezeichnung Ingenieur seien. Dieses Problem beschäftigte ebenso zeitgenössische Autoren wie den Hüttendirektor Schlink, der es in den 187oerJahren dementsprechend ablehnte, von einer einheitlichen Gruppe der Techniker zu sprechen. Hieraus leitete er die Notwendigkeit ab, dass jeder Vertreter der technischen Profession selbst für den eigenen Aufstieg und Anerkennung zu sorgen habe, da es keine für alle technischen Bereiche gültige einheitliche Standespolitik geben könne. ${ }^{16}$

Diese Definitionsproblematik greift auch die zeitgenössische Ratgeberliteratur auf. In der Heftreihe Was willst du werden?, die sich an Jugendliche richtete und diesen verschiedene Berufe näherbrachte, fühlte sich der Autor, Ingenieur Schotte, 1867 genötigt, im Band über den Beruf des Ingenieurs und Maschinentechnikers "trotz der großen Abweichungen der einzelnen Berufszweige unter einander" die "Vereinigungspunkte in der Thätigkeit“ hervorzuheben, um den Ingenieur dennoch einer einheitlichen Profession zuzuordnen. ${ }^{17}$ Gemeinsam wähnt er ihnen „das Interesse am öffentlichen Leben“, das "Studium der exacten Wissenschaften“, ihr gemeinsames Ziel der "Gestaltung von Materie“ sowie ihre Mittlerrolle in der "Arbeiterfrage“. 18 Münzinger pflichtete dieser Sicht bei, doch „im Gegensatz zu anderen Berufen wie Ärzten, Pfarrern oder Juristen" sieht er das verbindende Element in der Herausforderung, „daß Ingenieure praktisch-technische Probleme mit intellektuellen Mitteln zu lösen haben. ${ }^{19}$

Verbindend war auch das Geschlecht, da es sich bei den Ingenieuren fast ausschließlich um Männer handelte. Frauen konnten zwar seit dem ersten Jahrzehnt des 2o. Jahrhunderts an Technischen Hochschulen studieren, doch blieb ihre Zahl bis zum Ersten Weltkrieg marginal und erfuhr erst in der Zwischenkriegszeit einen nennenswerten Anstieg. ${ }^{20}$ Reinhold Jäckel legte 1908 mit seiner Statistik über die Lage der technischen Privatbeamten in Groß-Berlin eine erste statistische Untersuchung eines geografisch wie sozial begrenzten Ausschnitts der deutschen Technikerschaft vor. Für den untersuchten

\footnotetext{
15 Gispen, Ingenieurelite, 1994, S. 221; vgl. auch Klages /Hortleder, Gesellschaftsbild, 1994, S. 269 f.; Manegold, Akademisierung der Technik, 1981, S. 96 f.

16 Braun, Anschauungen von Ingenieuren, 1978, S. 223.

17 Schotte, Beruf des Ingenieurs, 1871, S. 8.

18 Ebda., S. 8 f.

19 Münzinger, Baumeister einer besseren Welt, 1947, S. 96.

20 König, Staatsdiener, 2006, S. 220; Zachmann, Mobilisierung der Frauen, 2004, S. 133-135.
} 
Ausschnitt konstatierte er eine Frauenquote von weniger als zwei Promille, wobei die Fachrichtung Chemie überproportional repräsentiert war. ${ }^{21}$ Der Bereich der Kolonialtechnik ist in diesem Sinne als gänzlich maskulin zu bezeichnen. In den Akten und Veröffentlichungen weist nichts darauf hin, dass es auch Technikerinnen oder Ingenieurinnen gab, die in den deutschen Kolonien beim Gouvernement oder bei Privatfirmen ihr Auskommen fanden. ${ }^{22}$

$\mathrm{Ob}$ aber die eingangs angesprochene Heterogenität derjenigen, die mit dem Prädikat Ingenieur oder Techniker versehen wurden, auch die Situation ihrer in den Schutzgebieten beschäftigten Kollegen widerspiegelte, soll später im Rahmen dieser Dissertation geprüft werden. Es ist jedoch eindeutig, dass jene Gruppe fast ausschließlich Ingenieure des Typus der Angestellten sowie der Staatsbeamten umfasste, wobei das Idealbild des Beamten - vor allem des Hoch- und Tiefbaus - vor dem Hintergrund des abnehmenden Prozentsatzes der Ingenieure, die hier ihr Auskommen fanden, zunehmend an Bedeutung verlor. ${ }^{23}$ Ebenso erging es dem Prototyp des selbstständigen Ingenieurunternehmers, der noch Ende des 19. Jahrhunderts eine Vorbild- und Idealfigur für die Berufsgruppe dargestellt hatte. Dieser büßte jedoch im Deutschen Reich massiv an Bedeutung ein und ist in den Quellen über die koloniale Arbeit nicht mehr zu greifen, mit Ausnahme einer sich in DSWA zeitweilig entwickelnden Schicht von Architekten und im Baugewerbe selbstständig tätigen Handwerkern. ${ }^{24}$

\section{Prestige der Ingenieure}

Ungeachtet der vielen unterschiedlichen Fachbereiche, aus denen sich die Gruppe der technischen Experten rekrutierte, teilten sie die Wahrnehmung der Geringschätzung der eigenen Profession durch prestigeträchtige gesellschaftliche Eliten. Zunächst soll daher auf diese Problematik abgehoben werden, da sich hieraus eventuelle Konsequenzen und Anreize für einen Übertritt der Ingenieure in den Kolonialdienst ableiten lassen. Gerade in den Darstellungen

21 Jäckel, Statistik, 19o8, S. 6 f.; vgl. dazu auch Scholl, der in zehn Bänden der Neuen Deutschen Biographie keinen Artikel findet, der sich auf eine Ingenieurin bezieht: Scholl, Ingenieur in Ausbildung, 1981, S. 27.

22 Vielmehr wurde der "Mangel“ an deutschen Frauen in den Schutzgebieten immer wieder als abzustellendes Übel beschrieben, vgl. hierzu u. a.: Kundrus, Moderne Imperialisten, 2003, S. 77-96; Lerp, Bevölkerungspolitik, 2009, S. 32-39.

23 Sander, Ingenieurwesen, 2008, S. 221.

24 Klages/Hortleder, Gesellschaftsbild, 1994, S. 274 f.; vgl. für DSWA: Peters, Baukunst in Südwestafrika, 1981. 
zur sozialen Stellung des technischen Experten durch den Verein Deutscher Ingenieure (VDI), als ältesten und bedeutendsten deutschen Ingenieursverein, bediente man sich gern der Äußerungen des Eisenbahningenieurs Max Maria von Weber, Sohn des berühmten Komponisten Carl Maria von Weber. Jener äußerte sich explizit zur Lage der deutschen Techniker und beschrieb die „Berufsclasse der Techniker“ als „Emporkömmling im Völkerleben“. ${ }^{25}$

Sehr eindrucksvoll verleiht er diesem Sachverhalt in einem imaginären Gespräch untervier Augen zwischen dem Grafen C und dem Baron E literarisch Ausdruck. In dem Gespräch gibt der Baron dem Grafen bekannt, dass sein Sohn - allseits gebildet und begabt - Techniker werden möchte. Dies ruft bei seinem Gegenüber nur Unverständnis und zum Teil gar Belustigung hervor. ${ }^{26}$ Im Verlauf des Gesprächs stellt Weber den Grafen als naiven Vertreter einer mit antitechnischen Ressentiments behafteten Elite dar. Dieser verweigert selbst einem Ingenieur, der in seinem Auftreten den betreffenden gesellschaftlichen Anforderungen entspricht und erfolgreich an einem Polytechnikum studiert hat, jegliche Anerkennung. Vielmehr beschwert sich ebenjener Graf über dessen scheinbar anmaßendes Verhalten: „Wie kann ein praktischer Techniker Alluren haben, sprechen und gehen und stehen und Damen grüßen wie unsereiner. - Lächerlich!“, um kurz darauf den sich anschließenden Fragen des Barons zu entgegnen:

Sie können sich kaum über seinen Beruf beim Anblick eines Schornsteinfegers, oder Müllers [...] täuschen, auch wenn er sich auf das Sauberste endimanchirt hat. Sie fühlen die Roture [das Nichtadligsein, S. B.], [...] wenn unser Herr Maschinist so, oder Herr Ingenieur so und so auch noch so brüsk und selbstbewußt durch den Salon tappt. ${ }^{27}$

Die auffallende Verknüpfung von Berufsstand und Habitus, die dem Techniker durch seine Arbeit ein bestimmtes Verhalten als ,natürlich' nahelegt, lässt jegliche Anpassung an die bessere Gesellschaft im Sinne der Übertretung sozialer Schranken als unangebracht, wenn nicht gar unmöglich erscheinen. Es ist durchaus bemerkenswert, dass der Baron solche Bedenken nicht hegt und selbst an der Berufswahl seines Sohnes nichts Ehrenrühriges oder Bedenkliches findet. Mit Bourdieu lässt sich Webers Geschichte wie eine Prognose des Kommenden lesen, als Parabel auf den Eintritt der Technik und ihrer Akteure in die prestigeträchtigen Kreise der Gesellschaft. Zwar fällt der Ingenieur in der gehobenen Gesellschaft noch auf, doch sind es gerade die selbstbewussten

\footnotetext{
25 Weber, Stellung der Deutschen Techniker, 1877, S. 5 [Herv. i. O.].

26 Weber, Wo steht der deutsche Techniker, 1882, S. 420.

27 Ebda., S. 432.
} 
Schritte, die als brüsk empfunden werden, die zeigen, wie selbstverständlich und bewusst er den noch fremden Raum der höheren Gesellschaft bereits für sich einnimmt und welche Stellung im sozialen wie physischen Raum er für sich beansprucht. ${ }^{28}$

Martin Schwarz bestätigt den Eindruck, den Weber im Rahmen seines imaginierten Dialogs von der sozialen Stellung der Ingenieure zeichnete, wenn er auf die Wahrnehmung dieser Gruppe als Parvenü verweist, die dem Handwerker nahestand. ${ }^{29}$ Die Profession dürfe daher bestenfalls auf Duldung, als auf wirkliche Anerkennung in der Gesellschaft hoffen. Immer wieder führten die betroffenen Ingenieure und Techniker England und Frankreich als beispielhaft an, da hier ihrer Profession nicht die Rolle des Emporkömmlings zugeschrieben wurde, der nach Möglichkeit aus dem gesellschaftlichen und staatlichen Leben fernzuhalten sei. ${ }^{30}$ Besonders die hochrangigen Positionen, die Techniker und Ingenieure im französischen Staatsapparat erreichen konnten, gaben immer wieder Anlass zu neidvollen Blicken über den Rhein. ${ }^{31}$

Für die vermeintliche soziale Missachtung der Ingenieure wurden vor allem zwei Gründe hervorgehoben: Zum einen beruhte die Wahrnehmung als Neuling auf ihrer relativ späten Entwicklung als eigenständige Berufsgruppe in den deutschen Staaten im Gegensatz zu Frankreich oder England. In Deutschland sei die Entwicklung der Profession der Ingenieure und Techniker vom Eisenbahnbau überrascht worden, der einen sprunghaft ansteigenden Bedarf an technisch gebildeten Kräften zur Folge gehabt habe. ${ }^{32}$ Weber gab hier zu bedenken, dass dieser Bedarf in der ersten Hälfte des 19. Jahrhunderts mit Personen bedient werden musste, die weder fachlich noch gesellschaftlich genügend gebildet waren. Sie errangen nun aber unter den gegebenen Umständen hohe Positionen beim Infrastrukturbau und kamen damit mit gesellschaftlich anerkannten Kreisen in Berührung. Diese frühen vor allem über die technische Praxis aufgestiegenen Personen prägten, so Weber, nun aber das Bild, das die bessere Gesellschaft von den Ingenieuren und damit auch von der Technik insgesamt hätte. ${ }^{33}$ Gleichwohl sah Weber die Ingenieure

28 Vgl. dazu Bourdieu, Die feinen Unterschiede, 2008, S. 739; Barlösius, Pierre Bourdieu, 2011, S. 38 .

29 Schwarz, Härte des Krieges, 2012, S. 8.

$30 \quad$ Weber, Stellung der deutschen Techniker, 1877, S. 5 f., 13.

31 Vgl. u. a. Manegold, Der VDI, 1981, S. 140.

32 Vgl. Radkau, Technik in Deutschland, 2008, S. 146.

33 Weber, Stellung der deutschen Techniker, 1877, S. 10-12. Scholl verweist für das Königreich Hannover ebenfalls auf einen sprunghaft angestiegenen Bedarf und erklärt damit die höhere Zahl an Söhnen von Arbeitern und niedrigen Beamten, die im Eisenbahnbau angestellt waren: Scholl, Ingenieure in der Frühindustrialisierung, 1978, S. 206 f. 
selbst in der Pflicht und bemängelte die fehlende Solidarität untereinander. ${ }^{34}$ Zudem ließ man sich von „Behörden“ und „Privatleuten“, die „sich einen vertrauenswürdigen praktischen Techniker nicht anders vorstellen können, als einen Mann ziemlich ordinären Exterieurs, in Wasserstiefeln, mit einem Zollstab unter'm Arme, einer Papierrolle in der Hand“, in eine Rolle zwingen. Dieses Verhalten sei auch dafür verantwortlich, dass der Ingenieur noch im ausgehenden 19. Jahrhundert nicht jene Stellung innehatte, die er Weber zufolge verdiente. ${ }^{35}$ Einen Hinweis auf diese Vorurteile gegenüber der technischen Profession gibt ein Brief Walther Rathenaus vom 19. Mai $1908 \mathrm{zu}$ Beginn seiner Reise nach DSWA. Unter seinen Reisegefährten befand sich auch der Bauinspektor Schlüpmann, der selbst über Jahre hinweg in den deutschen Kolonien gearbeitet hatte. Diesen beschrieb Rathenau kurz: „ein jüngerer Mann, sehr Techniker, aber durchaus akzeptabel im Umgang “. ${ }^{36}$

Eine zweite Ursache für das ablehnende Verhalten gewisser gesellschaftlicher Gruppen gegenüber den Ingenieuren meinte Weber zudem in der modernen Technik als solcher zu erkennen. Nicht nur verhinderte ihre Neuheit eine klare Einordnung der technischen Profession in eine der bestehenden Schichten, wie technische Fachzeitschriften bereits früh im 19. Jahrhundert bemerkten, auch unterstellte Weber den bestehenden Eliten einen neidvollen Blick auf die Technik hinsichtlich ihrer gesellschaftlichen Bedeutung. ${ }^{37}$ Der Dichteringenieur deutete dies als einen Ausdruck des Unbehagens gegenüber der immer gewichtigeren Rolle der Technik und damit auch ihrer Vertreter, durch die sich die etablierten Professionen bedroht sahen. ${ }^{38}$ Seine Äußerungen galten nicht nur den zeitgenössischen Ingenieuren und Technikern als treffende Diagnose ihrer sozialen Situation sowie abzustellender Missstände, sondern lieferten bis in die Gegenwart hinein die Diskussionsgrundlage für Technikhistoriker in Bezug auf die Stellung der technischen Profession in der Gesellschaft des ausgehenden 19. Jahrhunderts.

Webers zugegebenermaßen nicht sehr originelle These der wachsenden gesellschaftlichen Bedeutung der Technik findet sich später auch in der Studie von Gerd Hortleder aus dem Jahr 1973. In dieser vertritt der Soziologe die Auffassung, Ingenieure hätten daran geglaubt, dass mit steigender Bedeutung

34 Weber, Wo steht der deutsche Techniker, 1882, S. 426 f.; vgl. dazu auch Weber, Stellung der deutschen Techniker, 1877, S. 6, 20-22.

35 Weber, Wo steht der deutsche Techniker, 1882, S. 426.

36 Rathenau/Jaser, Walther-Rathenau-Gesamtausgabe, Nr. 5, 2006, S. 383 .

37 Vgl. Scholl, Ingenieure in der Frühindustrialisierung, 1978, S. 21 f.; Manegold, Akademisierung der Technik, 1981, S. 98-10o.

38 Weber, Stellung der deutschen Techniker, 1877, S. 5; vgl. auch Hänseroth, Technischer Fortschritt, 2013, S. 278. 
der Technik auch ihr gesellschaftliches Prestige anwachsen würde. „Diese Hoffnung hat sich aber nicht erfüllt.،"39 Und dies, obwohl es Ende des 19. Jahrhunderts auch in der bürgerlichen Gesellschaft keinen Zweifel mehr an den zumindest materiellen Errungenschaften und Verbesserungen durch Technik und Industrialisierung gab. ${ }^{40}$ Die Wahrnehmung vermeintlicher gesellschaftlicher Missachtung wäre demnach vor allem die Folge der Erwartungsdifferenz zwischen selbst zugeschriebener Bedeutung und tatsächlich erhaltener Anerkennung.

Die diesbezüglichen Äußerungen von Ingenieuren und Technikern lassen ein klares argumentatives Muster erkennen. Zum einen verknüpften sie das allgemeine Wohl der Bevölkerung mit dem technischen Fortschritt. Diesem kam damit eine enorme Bedeutung zu. Eine Hochschätzung der Technik bedeutete aber auch einen Ansehensgewinn für ihre Akteure. Thesenhaft verkürzt hieß das: „Jeder Mangel an Geltung der Technik im öffentlichen Leben schädigt [...] vor allem die Allgemeinheit, weil er die Möglichkeiten der Technik beschränkt, der Allgemeinheit zu dienen. ${ }^{41}$ Diese der Technik zugesprochene Gemeinsinnsorientierung adaptierten die Ingenieure und Techniker für ihre Berufsgruppe. Ganz ähnlich war in einer Ende des 19. Jahrhunderts und am Beginn des 2o. Jahrhunderts in neun Auflagen erschienenen Broschüre zum Berufseinstieg junger Männer über die Profession des Maschineningenieurs zu lesen: „In seiner Hand liegt die Anwendung der Wissenschaft auf das praktische Leben zum Segen der Menschheit.“42 Denn bei dieser Profession handele es sich „im eigentlichsten Sinne [um] ein[en] Beruf der Gegenwart und noch mehr vielleicht der Zukunft."43 Verweise auf eine möglicherweise ungenügende soziale Stellung der Ingenieure und Techniker oder fehlendes Prestige ihres Berufszweiges fehlen in dieser Schrift jedoch gänzlich.

Die Rede von Conrad Matschoß, dem langjährigen Professor an der TH Charlottenburg, zum fünfzigjährigen Jubiläum des VDI Bezirksvereins in Köln, in der er 1911 Zur Bedeutung des Ingenieurs sprach, setzte dementgegen einen anderen Schwerpunkt. Die großen Errungenschaften der Technik, die wir "heute so staunend bewundern“, waren seines Erachtens nur „mit rücksichtsloser Hintansetzung von dem, was das Leben sonst an Freuden und

39 Hortleder, Ingenieure in der Industriegesellschaft, 1973, S. 17; vgl. auch Scholl, Ingenieur in Ausbildung, 1981, S. 57 .

40 Rohkrämer, Eine andere Moderne?, 1999, S. 111; Fraunholz/Wölfel, Hochmoderne Ingenieure, 2012, S. 23-25.

41 Anonymus, Betreff Vorschlag Wangelin, 1919, Anlage 1.

42 Anonymus, Maschineningenieur und Maschinentechniker, ca. 1895, S. 3.

43 Ebda. 
Erholung bietet, gepackt von der Liebe zum Beruf" zu erreichen. ${ }^{44}$ Auch an anderer Stelle suchte Matschoß, den „Männern der Technik“ ein Denkmal zu setzen, das sie bisher trotz ihrer Verdienste und Aufopferung für die Gesellschaft nicht bekommen hätten. So konstatierte er im Vorwort seiner pünktlich zur Eröffnung des Deutschen Museums 1925 fertiggestellten Sammlung von Biografien bedeutender Techniker: „Die Männer der Technik hat der Ruhm noch nie verwöhnt.“45 Doch nicht nur die Bedeutung der Technik sollte sich in Form von Anerkennung auf die technische Profession übertragen, auch die Argumentationsmuster, die die Technik auf das Podest einer Kulturleistung hoben, dienten jenem Zweck. ${ }^{46}$ Dementsprechend begannen Ingenieure im 19. Jahrhundert, den eigenen sozialen Status über den Umweg der Selbstdarstellung als Kulturpioniere zu erhöhen. Gegen eine Wahrnehmung als etwas Akulturelles argumentierten sie mit der kulturschaffenden oder zumindest kulturermöglichenden Wirkung von Technik. Nachdem der formalen Gleichsetzung der technisch-akademischen Ausbildung mit den Universitätsabschlüssen der bestehenden Eliten keine gesellschaftliche Gleichstellung der Ingenieure und Techniker gefolgt war, bediente man sich verstärkt der beschriebenen Argumentationsmuster. ${ }^{47}$

Die von der technischen Profession immer wieder beschworene Bedeutung der Technik für alle Lebensbereiche einer modernen industrialisierten Gesellschaft schlug mit der Niederlage im Ersten Weltkrieg in ihr Gegenteil um. ${ }^{48}$ Nun wurde gerade jener Berufsgruppe eine bedeutende Mitschuld am verlorenen Krieg zugeschrieben. ${ }^{49}$ Graf Posadowsky, Mitglied der Deutschnationalen Volkspartei, vertrat die Position, „[w]enn wir den Krieg verloren haben, so sind wir nicht militärisch besiegt worden[,] sondern technisch“".50 Die von publizierenden Ingenieuren des VDI wie des Reichsbundes Deutscher Technik (RDT) als „technizistische Dolchstoßlegende“ diffamierte Behauptung wurde von selbigen argumentativ gegen den "Militarismus" gewendet, der "der deutschen Technik in den Arm gefallen ist. ${ }^{\text {“1 }}$ Demzufolge hatte nicht ein Versagen der verantwortlichen Techniker zur Niederlage im Krieg geführt, sondern vielmehr die Ignoranz der Militärs. Solche Vorhaltungen bestärkten

\footnotetext{
44 Anonymus, Bedeutung des Ingenieurs, 1911.

45 Matschoß, Männer der Technik, 1925.

46 Manegold, Der VDI, 1981, S. $139 \mathrm{f}$.

47 Vgl. Dietz u. a., Kulturwert der Technik, 1996, S. 4 f.

48 Scholl, Ingenieur in Ausbildung, 1981, S. 57.

49 Dietz u. a., Kulturwert der Technik, 1996, S. 6 f.

$5^{\circ}$ Anonymus, Ausgang des Krieges, 1919.

51 Ebda.; vgl. dazu Viefhaus, Ingenieure in der Weimarer Republik, 1981, S. 292 f.; Radkau, Technik in Deutschland, 2008, S. 255 f.
} 
bei der technischen Profession das Gefühl der unangemessenen Behandlung sowie ihrer gesellschaftlichen Zurückweisung. Diesen standen nun Vertreter der antitechnischen Kulturkritik vor allem aus dem Bereich der Geisteswissenschaften gegenüber, die man vonseiten der Ingenieure durch den Verweis auf die kulturschaffende Wirkung der Technik zu entkräften versuchte. ${ }^{52}$ Es waren jedoch gerade jene „Kulturkritiker“, die mit Bezug auf die entscheidende Rolle der Technik im Krieg ihre ablehnende Haltung gegenüber den Ingenieuren bestätigt sahen..$^{53}$ Allgemein konstatieren Technikhistoriker für die ersten Jahrzehnte des 2o. Jahrhunderts jedoch einen positiven Blick der deutschen Gesellschaft auf Industrie und technischen Fortschritt. ${ }^{54}$

Aus dem bisher Gesagten lässt sich bereits die Illusio des Feldes der Technik ableiten, die von den Vertretern der technischen Profession geteilt wird, die sich auf das Spiel im betreffenden Feld einlassen. Verbindend ist der gemeinsame Glaube an den technischen Fortschritt, der ausnahmslos positiv bewertet, häufig sogar mit gesellschaftlichem Fortschritt gleichgesetzt wird. Dieser Glaube ist als Nomos des technischen Feldes zu verstehen und damit als grundlegende Prämisse, die dem feldspezifischen Handeln der beteiligten Akteure zugrunde liegt. Wie bereits dargelegt, galt dieser Nomos auch im kolonialen Subbereich. Die Orientierung am Gemeinwohl beziehungsweise dem, was man dafür hielt, verbunden mit der Bereitschaft, sich dem Erreichen der bestmöglichen Lösung unterzuordnen, kann ebenso als wirkmächtige Illusio verstanden werden. ${ }^{55}$ Das Selbstbild dieser Gruppe war auf der einen Seite geprägt von einer als ungerecht empfundenen sozialen Herabstufung bei gleichzeitiger Betonung des eigenen neutralen und nur der Objektivität verpflichteten Handelns. Hortleder charakterisiert dieses Verhältnis zwischen Ingenieur und Staat gar als „einseitige Liebe, über die der Ingenieur umso mehr verbittert sein mußte, als er nichts weiter wünschte, als seine Kraft und Energie ganz ,in den Dienst' dieses Staates stellen zu dürfen."56 Eine Maßgabe, die sich vermeintlich zwingend aus den Besonderheiten der technisch konstruktiven Arbeit ergab. Damit ging aber auch eine gewisse Ablehnung der Verantwortung für die gesellschaftlichen oder sozialen Folgen des eigenen Handelns einher, für die die Gruppe der Techniker nicht oder nur bedingt

\footnotetext{
52 Vgl. Walle, Technikrezeption, 1994, S. 101; Hortleder, Gesellschaftsbild, 1970, S. 85 f. Zur Gegenargumentation: Dietz u. a., Kulturwert der Technik, 1996, S. 7.

53 Hortleder, Gesellschaftsbild, 1970, S. 79 f.

54 Rohkrämer, Eine andere Moderne?, 1999, S. 39; Fraunholz/Wölfel, Hochmoderne Ingenieure, 2012, S. 23 .

55 Vgl. Hortleder, Gesellschaftsbild, 1970, S. 75 f.; dazu allgemein Hardensett, Der Technische Mensch, 1932.

$5^{6}$ Hortleder, Gesellschaftsbild, 1970, S. 72.
} 
einstehen wollte. ${ }^{57}$ Eine Haltung, die sich in dem zu einer gewissen Bekanntheit gelangten Zitat aus den Erinnerungen Albert Speers ausdrückte und pointiert den Titel des Sammelbandes einer Ausstellung des Museums für Technik und Verkehr in Berlin gab: „Ich diente nur der Technik“.58

Die radikalste Form der technischen Vorstellung einer Ausrichtung am Gemeinwohl ist die Idee der Technokratie. ${ }^{59}$ War für Hortleder die Idee des Gemeinwohls ein Versuch, ,in den politischen Raum einzudringen, ohne Politik zu treiben“, ${ }^{60}$ so wurde die Herrschaft der Technik(er) zur Ultima Ratio gewisser technischer Kreise, die das Heil in einer Staatsform sahen, die sich vollkommen an technischen Gegebenheiten und Zweckrationalität orientierte und damit klassisches politisches Handeln als obsolet betrachtete. ${ }^{61}$ Obwohl diese Bewegung selbst unter den Ingenieuren und Technikern nur eine Randerscheinung blieb, ist sie doch für die postkoloniale Arbeit der Kolonialingenieure von großer Bedeutung. Innerhalb des RDT fanden solche politischen Äußerungen mehr Gewicht. Ihre Forderungen reichten von der gezielten Wahl politischer Kandidaten, die selbst Techniker oder Ingenieure waren, bis hin zu radikalen Forderungen, die eine vollständige Umgestaltung des Staates nach technisch-objektiven Gesichtspunkten befürworteten. Begleitet wurden solche Vorschläge jedoch auch immer wieder von selbstkritischen Vorwürfen, sich aktiv aus politischen und gesellschaftlichen Diskussionen fernzuhalten. ${ }^{62}$ Dies führte man noch in den 198oer-Jahren auf den Umstand zurück, zuvor bei politischen Entscheidungen vollständig übergangen worden zu sein und mit den eigenen Forderungen kein Gehör gefunden zu haben. ${ }^{63}$ Ebenso sah Karl-Heinz Manegold in der geringen Zahl von Parlamentariern - zwischen 1887 und 1912 waren es zeitgleich nie mehr als zwei Vertreter der technischen Profession - einen Grund für diese apolitische Haltung. ${ }^{64}$

57 Dietz u. a., Kulturwert der Technik, 1996, S. 3; Hortleder, Ingenieure in der Industriegesellschaft, 1973, S. 18.

$5^{8}$ Gottwaldt/Klewin, Ich diente nur der Technik, 1995. Im Original lautet das Zitat: „Entscheidende Jahre meines Lebens habe ich der Technik gedient, geblendet von ihren Möglichkeiten. Am Ende, ihr gegenüber, steht Skepsis“: Speer, Erinnerungen, 1976, S. 525.

59 Auf den Zusammenhang von wahrgenommener gesellschaftlicher Missachtung und einer Tendenz zu technokratischen Ideen bei den deutschen Ingenieuren verweist bereits Manegold, Emanzipation der Technik, 1977, S. 5 o.

6o Hortleder, Gesellschaftsbild, 1970, S. 76.

61 Vgl. Dietz u. a., Kulturwert der Technik, 1996, S. 8.

62 Ebda., S. 3 f.

63 Vgl. Scholl, Ingenieur in Ausbildung, 1981, S. 57.

64 Manegold, Der VDI, 1981, S. 140. 
Essenziell für die Genese eines Feldes wie ebenjenem der Technik ist dessen Fähigkeit zur Reproduktion sowie eine gewisse Autonomie. ${ }^{65}$ Diese ist aber nie vollkommen, da sich die Felder gegenseitig beeinflussen. Im Folgenden wird der Schwerpunkt der Betrachtung auf das System der akademischen Ausbildung der Ingenieure im ausgehenden 19. Jahrhundert gelegt und dabei überprüft, welchen sozialen Schichten die betreffenden Personen angehörten und welche Konsequenzen die zunehmende Formalisierung der technischen Ausbildung hatte.

Lassen sich andere Berufsgruppen wie Ärzte hinsichtlich formalisierter Abschlüsse und Bildungspatente recht klar von Laien abgrenzen, so trifft dies im frühen 19. Jahrhundert auf Ingenieure nicht zu. Die noch zu beschreibende Entwicklung hin zu einheitlichen und für alle Techniker und Ingenieure verpflichtenden Ausbildungswegen und Examina diente neben der Befestigung eines gewissen Bildungsideals auch der Abgrenzung der Ingenieursgruppe nach außen, im Zusammenhang mit einer Binnendifferenzierung. Die formale akademische Ausbildung samt der zu erwerbenden Bildungstitel stellte im 19. Jahrhundert auch auf dem europäischen Kontinent lediglich eine von mehreren Möglichkeiten dar, eine Tätigkeit als Ingenieur oder Techniker wahrzunehmen. Wenig einheitlich strukturierte praktische Ausbildungswege blieben bis ins beginnende 20. Jahrhundert hinein eine Quelle der Ingenieurbildung und eine Ursache für Auseinandersetzungen. ${ }^{66}$ Bis in die 188oer-Jahre hinein sprach man in der Öffentlichkeit generalisierend von Technikern, meist ohne sprachliche Differenzierung. Dies wertet Sander als Indiz für den prekären sozialen Status, den die Ingenieure bis ins 20. Jahrhundert hinein behalten sollten. ${ }^{67}$ Diese Praxis schlug sich auch in den Schreiben der jeweiligen kolonialen Gouvernements sowie des RKA nieder, indem Begriffe wie Techniker oder Ingenieur bis zum Ersten Weltkrieg weitgehend synonym gebraucht wurden. Lediglich bei höheren akademischen Abschlüssen und Stellenbezeichnungen wie Regierungsbaumeister oder Eisenbahnkommissar erfolgte eine Differenzierung.

Die Möglichkeit der reinen praktischen Ausbildung beschränkte sich in der zweiten Hälfte des 19. Jahrhunderts jedoch zunehmend auf einzelne

65 Kieserling, Felder und Klassen, 2008, S. 6 f.

66 Auf dieses „Problem der Grenzen“ weist Bourdieu für andere Felder hin, in denen immer wieder Kämpfe um die Zugehörigkeit zum Feld ausgetragen werden: Bourdieu, Rede und Antwort, 1992, S. 159 f.

67 Sander, Ingenieurwesen, 2008, S. 23 . 
Techniker, die sich als Autodidakten das nötige fachliche und praktische Wissen angeeignet hatten. In Jäckels Studie über die technischen Privatbeamten in Berlin traf dies gerade einmal noch auf fünf Prozent der Befragten zu. Ein Hoch- oder Fachschulabschluss war im deutschen Raum für höhere technische Berufe zur Regel geworden. ${ }^{68}$ Der Erwerb von staatlich anerkannten Bildungspatenten, also institutionalisiertem kulturellem Kapital, stieg damit zum entscheidenden gatekeeper für eine zukünftige berufliche Stellung und Position im technischen Feld auf und löste inkorporiertes kulturelles Kapital als entscheidenden Faktor ab. ${ }^{69}$ Dieses wurde in der praktischen Ausübung eines technischen Berufes erworben und bedurfte im Gegensatz zu Bildungstiteln des wiederholten Nachweises in der Praxis. ${ }^{70}$ Im Grunde folgte man mit dieser Institutionalisierung der technischen Ausbildung der französischen Entwicklung. Hier hatte sich bereits im Verlauf des 18. Jahrhunderts ein nach militärischen und zivilen Aufgaben differenzierter Ingenieursstand herausgebildet, der sich vergleichsweise deutlich durch den Besuch und Abschluss entweder der école du génie militaire oder der école des ponts et chaussées unterschied, die 1748 und 1747 gegründet worden waren. ${ }^{71}$ Da beide Schulen als Institutionen des Ancien Régime galten, wurden sie während der Französischen Revolution vorübergehend geschlossen. Stattdessen wurde der Besuch der 1794 in Paris gegründeten école polytechnique für Ingenieure, die eine Anstellung im Staatsdienst anstrebten, obligatorisch. ${ }^{72}$

Idealtypisch werden mit der Institutionalisierung des technischen Zugangsund Bildungswesens im Deutschen Reich zwei Arten der Ingenieurbildung in Verbindung gebracht: die von David Noble beschriebene, eigentlich auf den amerikanischen Kontext bezogene "school culture“ sowie die „shop“ beziehungsweise „field culture“. Auf dem nordamerikanischen Kontinent entwickelte sich infolge des hohen Bedarfs an technisch gebildeten Kräften für nationale Infrastrukturprojekte eine „school culture“, die für eine formalisierte und verschulte Form der Ingenieurbildung steht. Auch hier legten die Ausbildungsstätten ihren Schwerpunkt auf mathematisches und theoretisches Wissen. Die älteren in der Praxis geschulten Kohorten dominierten in Industrie und Bauwirtschaft und nahmen bessere Positionen ein als ihre Kollegen aus

68 Vgl. Jäckel, Statistik, 1908, S. 43; Kohlrausch/Trischler, Building Europe, 2014, S. 40; König, Staatsdiener, 2006, S. 200 f.

69 Vgl. Krais, Einleitung, 1981, S. 8.

70 Bourdieu, Ökonomisches Kapital (Mechanismen der Macht), 1992, S. 61 f.

71 Vgl. Scholl, Ingenieure in der Frühindustrialisierung, 1978, S. 15 f.; König, Staatsdiener, 2006, v. a. S. 179-187.

72 Ebda., S. $181 \mathrm{f}$. 
den Bildungsinstitutionen. ${ }^{73}$ Der Konflikt zwischen diesen beiden Gruppen ist unschwer als Machtkampf im betreffenden Feld der Technik in den USA zu identifizieren. Die Illusio, also der Glaube an den Sinn des Spiels, blieb dabei unangetastet, Diskussionen entspannen sich nur infolge des Bedarfs an technischen Experten für den Staatsdienst um die Frage der angemessenen Art der technischen Bildung. ${ }^{74}$

Deutlich länger als die USA hielt das Ursprungsland der Industriellen Revolution am hergebrachten System der praktischen Ausbildung von Technikern und Ingenieuren im Rahmen einer persönlichen MeisterLehrling-Beziehung fest. Eine entscheidende Ursache für die Unterschiede im technischen Bildungswesen zwischen Großbritannien und dem späteren Deutschen Reich kann in der Intervention der politischen Machthaber gesehen werden, unter der Maßgabe, die eigene ökonomische Situation wie auch Machtposition zu verbessern. Zusätzlich verweist Kocka auf die besondere Notwendigkeit der Beamtenausbildung innerhalb eines zentralistischen Staatsapparates, die sich auch in den frühen Ansätzen der Volksbildung und im verhältnismäßig gut entwickelten höheren Schulwesen wiederfand und allgemein mit der der Industrialisierung zeitlich vorangegangenen Entwicklung einer öffentlichen Bürokratie begründet werden kann. ${ }^{75}$ Zusammenfassend führt er also drei grundlegende Ursachen für die Durchsetzung eines Berechtigungswesens in der technischen Ausbildung und damit auch des legitimierenden institutionalisierten Kulturkapitals an: Zum ersten sei dies in der bürokratischen Tradition der zentralistisch organisierten Staatsgebilde begründet, besonders - zweitens - im Zusammenspiel mit einer nachholenden Industrialisierung und drittens einer vor allem vom deutschen Bürgertum ausgehenden Wertschätzung von Bildung, samt der hierfür zuständigen staatlichen Einrichtungen, die in Großbritannien nicht in gleicher Weise „sozial legitimiert" waren. ${ }^{76}$

Das Feld der Technik entwickelte sich in Deutschland aus dem sozialen Feld heraus, dessen Wertvorstellungen es übernahm. Dementsprechend forderte auch der Ingenieur Schotte in seinem im Jahr der Reichsgründung erschienenen Heft über den Beruf des Ingenieurs, selbiger müsse nach seinem schulischen Abschluss durchgebildet sein, um den an ihn persönlich, aber

73 Vgl. Noble, America by Design, 1977, S. 26-28; auch Bourdieu/Boltanski, Titel und Stelle, 1981, S. 100.

74 Vgl. Noble, America by Design, 1977, S. 20-29; Lundgreen, Engineering Education, 1990, S. 33; Gispen, Gefesselte Prometheus, 20o6, S. 165-168.

75 Kocka, Die Angestellten, 1981, S. 92. Damit ließe sich auch die frühe Etablierung der school-culture im technischen Bildungswesen in Frankreich erklären.

76 Ebda., S. 93 . 
auch an seine Profession gerichteten Ansprüchen genügen zu können. ${ }^{77}$ Kulturelles Kapital umfasste damit nicht nur den Besitz technischen Spezialwissens und die Fähigkeit, technische Anlagen planen und bedienen zu können, sondern auch Allgemeinwissen im Rahmen eines bürgerlichen Bildungskanons. Diese Erweiterung der notwendigen Wissensbestände ist als Teil der Emanzipationsbestrebungen der Ingenieure und Techniker zu verstehen. Damit sollte der empfundenen Ungleichbehandlung im Vergleich zu anderen gesellschaftlichen Gruppen wie dem Adel, dem Bildungsbürgertum oder dem Militär, also den traditionellen Eliten der deutschen Staaten, entgegengewirkt werden. ${ }^{78}$

Ausgangspunkt dieser Entwicklungen waren die in den 1820er-Jahren in zahlreichen deutschen Staaten gegründeten technischen und Gewerbeschulen, wobei in der ersten Hälfte des 19. Jahrhunderts die Schulbezeichnungen variierten, was eine trennscharfe Kategorisierung erschwert. Auch die vermittelten Inhalte wiesen je nach Institution eine große Varianz auf. ${ }^{79}$ Zwei miteinander verwobene Entwicklungen dieser Epoche stechen deutlich heraus: zum einen die sukzessive Erhöhung der Eintrittsvoraussetzungen bei zunehmender Theoretisierung und Mathematisierung der vermittelten Inhalte zulasten praktischer Aspekte. ${ }^{80}$ Gerade jene höheren Lehranstalten machten in den Jahren nach der Reichsgründung das Abitur zur Voraussetzung. Dies kann als Ausweis der Zugehörigkeit zum Bildungsbürgertum verstanden werden, somit sollte das Sozialprestige der Gruppe der Ingenieure über den Umweg anerkannter Bildungspatente erhöht werden. ${ }^{81}$ Wenn Wolfgang König diese Entwicklung vor allem aus standespolitischer Sicht zu erklären sucht, so ist dieser Deutung entgegenzuhalten, dass die Ansprüche vonseiten der akademischen Ingenieure ebenso mit dem hohen Anteil bürgerlicher Söhne in ihren Reihen zu erklären ist. ${ }^{82}$ Mein Erklärungsansatz hebt darauf ab, dass mit dem verstärkten Eintritt bürgerlicher Schichten, diese auch ihre Anspruchshaltung hinsichtlich gesellschaftlicher Anerkennung einbrachten. Gleichzeitig wurde das bis dahin verbreitete Stipendienwesen, das es ökonomisch weniger gut ausgestatteten Schichten erlaubt hatte, ein technisches Studium

\footnotetext{
77 Schotte, Beruf des Ingenieurs, 1871, S. 14-16.

78 Dietz u. a., Kulturwert der Technik, 1996, S. 1.

79 Vgl. Treue, Gesellschafts- und Berufsgeschichte, 1978, S. 31.

$80 \quad$ Schmoller, Social- und Gewerbepolitik, 189o, S. 259-261.

81 Scholl, Ingenieur in Ausbildung, 1981, S. 58; vgl. dazu auch Weber, Wo steht der deutsche Techniker, 1882, S. 425; Manegold, Akademisierung der Technik, 1981, S. 109-111; Jarausch, Unfree Profession, 199o, S. 219.

82 König, Verein Deutscher Ingenieure, 1994, S. 306 f.
} 
aufzunehmen, weitgehend abgeschafft. ${ }^{83}$ Stattdessen führten die späteren Akademien, dann Technischen Hochschulen, ein Schulgeld ein. Solche Bildungsinstitutionen wurden nun für Handwerker, die lediglich über eine Volksschulbildung verfügten, schwerer erreichbar. Ein zweiter Entwicklungsstrang kennzeichnete die Institute und Gewerbeschulen, die in der zweiten Hälfte des 19. Jahrhunderts neben den praktisch-technischen Lehrinhalten auch solche aus den neu-humanistischen Gymnasien aufnahmen und sich zu Realschulen und -gymnasien ausformten. ${ }^{84}$

Als Ursache dieser Entwicklung führt Kocka neben funktional-ökonomischen Argumenten, wie dem tatsächlichen Bedarf bestimmter Industrien nach einer besseren wissenschaftlichen Ausbildung ihrer Bewerber, vor allem standespolitische Interessen einer sich herausbildenden technischen Intelligenz an. Diese versuchte sich mit solchen zusätzlichen Zugangsschranken, als eigenständige Profession gegenüber Handwerkern und Arbeitern zu etablieren und sozialen Aufstieg zu realisieren. ${ }^{85}$ Die entscheidende Frage nach Ursache und Wirkung dieser Entwicklung bleibt Kocka dennoch schuldig: Gelang es also der Berufsgruppe der Ingenieure durch veränderte Curricula und der Verwissenschaftlichung der Ausbildung, ihren gesellschaftlichen Stellenwert, im Sinne der Schichten, die ein technisches Studium anstrebten, zu erhöhen oder waren diese Veränderungen nicht vielmehr Anpassungen in der Folge des Zustroms ebensolcher bürgerlicher Schichten? Also der Söhne, die jener bürgerlichen Klientel entstammten, deren Ansehen und Wertschätzung die junge technische Profession zu gewinnen suchte?

Betont Kocka vor allem den Willen und die Möglichkeit, durch eine Anpassung der Lehrinhalte an humanistische Traditionen wie auch durch eine als Mathematisierung begriffene Verwissenschaftlichung den eigenen Status zu erhöhen, so weisen die statistischen Erhebungen über die soziale Zusammensetzung der Studierenden der Technischen Hochschulen in eine andere Richtung. ${ }^{86}$ Aussagen wie jene des Bundes technischer Angestellter und Beamter, der die fehlende soziale Anerkennung der gesamten technischen Profession seitens der bürgerlichen Eliten anmahnt, die ihren „Söhnen“ nicht erlaubten, den Beruf des Ingenieurs oder Technikers zu ergreifen, beweisen

83 Hingegen konnten für den Besuch von Technischen Fachschulen in Preußen seit 1901 Staatsstipendien vergeben und das Schulgeld erlassen werden, was auch der VDIForderung nach mehr sozialer Mobilität entgegenkam: Schütte, Technisches Bildungswesen, 2003, S. 49-52.

84 Vgl. Schmoller, Social- und Gewerbepolitik, 189o, S. 248; Kocka, Die Angestellten, 1981, S. $94 \mathrm{f}$.

85 Ebda., S. 95 .

86 Vgl. Manegold, Emanzipation der Technik, 1977, S. 37; Kocka, Die Angestellten, 1981, S. 97 f. 
die Langlebigkeit dieser Vorwürfe bis in die 1920er-Jahre hinein. ${ }^{87}$ Die Sozialstruktur der TH-Studenten sowie der entsprechenden Alumni lässt an solchen Äußerungen jedoch zweifeln. In diesem Sinne sind auch Zachmanns Aussagen $\mathrm{zu}$ verstehen, die die Tendenzen hin zu einer Verwissenschaftlichung der Ingenieurausbildung an den Technischen Hochschulen als Homogenisierungsbestrebungen deuten. ${ }^{88}$

Die eingangs bereits angeführte statistische Untersuchung von Jäckel aus dem Jahr 1908 verweist auf die Problematik, die Zugehörigkeit von Ingenieuren oder Technikern zu einer sozialen Schicht nachzuweisen. Jäckel zieht dafür zum einen die Bildung der Akteure sowie den Beruf der Eltern - fast ausschließlich des Vaters - heran. Bei seiner Untersuchung der technischen Privatbeamten in Berlin für das erste Jahrzehnt des 20. Jahrhunderts stellte er fest, dass die Nachkommen von Kaufleuten oder Privatbeamten gut ein Drittel dieser Berufsgruppe ausmachten. ${ }^{89}$ Übereinstimmend weist Scholl für den hannoverschen technischen Staatsdienst einen Anteil von 50 Prozent nach, der dem höheren und mittleren Beamtentum entstammte. Für den Eisenbahnbau im Königreich Hannover traf dies immerhin auf 40 Prozent zu. Diese Zahlen korrespondieren auch mit Jäckels Befunden, der für gut zwei Drittel aller technischen Beamten eine Zugehörigkeit zum oberen beziehungsweise zum Mittelstand nachwies. ${ }^{90}$

Für die vorliegende Untersuchung weitaus interessanter ist der Blick auf den Anteil der Studierenden, die sich aus dem Bereich der technischen Privatbeamten rekrutierten, dieser lag bei einem Sechstel, Handwerker und kleine Gewerbetreibende folgten mit zusammen ebenfalls knapp sechzehn Prozent. Die Nachkommen von Arbeitern waren mit acht Prozent nur halb so oft vertreten. ${ }^{91}$ Für das Jahr 1907 lassen sich dann unter Einbezug auch der kleineren Gewerbetreibenden mehr als die Hälfte der Ingenieure, die ein Studium an der TH abschlossen, einem bürgerlichen Elternhaus zuordnen. ${ }^{92}$ Mit dem

87 Vorstand des Bundes der technischen Angestellten und Beamten, 25 Jahre Technikergewerkschaft, 1929, S. $10 \mathrm{f}$.

88 Zachmann spricht davon, dass der erste Vorsitzende des VDI, Franz Grashof, mit seiner Politik auf die Ausgrenzung nicht bürgerlicher Schichten von der höheren technischen Bildung abzielte: Zachmann, Mobilisierung der Frauen, 2004, S. 120 f.

89 Jäckel, Statistik, 1908, S. 25 f.

9o Scholl, Ingenieure in der Frühindustrialisierung, 1978, S. 206, 219; Jäckel, Statistik, 19o8, S. $28 \mathrm{f}$.

91 Ebda., S. 25 f.

92 König spricht hier allgemeiner vom „Wirtschaftsbürgertum“: König, Staatsdiener, 20o6, S. 204; Sander, Ingenieurwesen, 2008, S. 230, 240. Auch Jarausch gibt an, dass mehr als ein Fünftel aller Ingenieure mit Hochschulbildung einem akademischen Elternhaus entstammte: Jarausch, Unfree Profession, 199o, S. 19, 234. 
Besuch einer akademischen Lehreinrichtung stieg die Wahrscheinlichkeit für den Nachwuchs, seinerseits eine entsprechende Position hinsichtlich des Verdienstes und der gesellschaftlichen Stellung zu erreichen. Dies legen auch die Zahlen für jene Gruppe nahe, deren Väter in der Kategorie Arbeiter zusammengefasst wurden. Diese waren unter den technischen Studierenden lediglich mit knapp fünf Prozent vertreten. Insgesamt entstammten nur vierzehn Prozent aller Hochschulabsolventen den unteren Ständen oder waren gar nicht zuzuordnen. ${ }^{93}$

Bereits an diesem Punkt lässt sich feststellen, dass es sich bei der Aufnahme auch eines technischen Studiums um eine nicht zu verachtende Eintrittsbarriere handelte. Um die Ausgaben für diese Ausbildung und weitere Kosten für Unterbringung und Versorgung der Studierenden zu decken, bedurfte es eines entsprechenden Einkommens oder Vermögens der Familie. Wie die zuvor angeführten Zahlen belegen, erschwerte dieser Umstand gerade Anwärtern aus dem Arbeitermilieu den Zugang zu höherer technischer Bildung. ${ }^{94}$ Diese Annahme stützt auch der mit 1,3 Prozent sehr geringe Anteil der Arbeiterschaft unter den akademisch-technisch Gebildeten in Berlin im ersten Jahrzehnt des 20. Jahrhunderts, deren Quote bei den Mittel- und Fachschulen mit über einem Zehntel fast achtfach höher lag. ${ }^{95}$

Der Umstand, dass ökonomisches Kapital und Zeit investiert werden musste, um den Regierungsbaumeister-Titel zu erhalten, lässt sich mit einer besseren Stellung, Ansehen und einem höheren Einkommen erklären. Dies entspräche einer gewissen Dividende für das eingesetzte Kapital. ${ }^{96}$ Solche „Bildungsinvestitionen" verschlangen also einen Teil des elterlichen Einkommens, das damit dem sofortigen Konsum entzogen wurde. Diese Annahme stützend spricht auch Hans Speier von einem "gewissen Wohlstand der Eltern“, der nötig sei, um ein technisches Studium zu finanzieren. ${ }^{97}$ Vorteile eines zeitlich näher liegenden Konsums schoben diese Elternhäuser zugunsten einer späteren Rendite ihrer Investitionen in die Ausbildung ihrer Kinder auf. ${ }^{98}$ Dass sich die Investition von Zeit und Geld jedoch auszahlte, war keineswegs sicher, sondern immer mit der Möglichkeit des Scheiterns verbunden, wenn beispielsweise das Diplom aufgrund schlechter Leistungen nicht erlangt wurde. Trotz dieser Unwägbarkeiten ist anzunehmen, dass jene Eltern, die bereit und in der Lage waren, ihren Nachkommen ein technisches Studium zu ermöglichen, dies

93 Vgl. Jäckel, Statistik, 19o8, S. 26.

94 Kocka, Die Angestellten, 1981, S. 101.

95 Jäckel, Statistik, 1908, S. 240.

96 Vgl. Krais, Einleitung, 1981, S. 8.

97 Speier, Die Angestellten vor dem Nationalsozialismus, 1977, S. 108.

98 Bourdieu, Die feinen Unterschiede, 2008, S. 520, 527. 
nicht als sozialen Abstieg verstanden. Vielmehr ist anzunehmen, dass sie davon ausgingen, dass sich ihre Investition sowohl auf der finanziellen als auch auf der Ebene gesellschaftlicher Anerkennung lohnen werde. ${ }^{99}$ Der Vorteil eines technischen Studiums, so liegt nahe, beschränkte sich demgemäß eben nicht nur auf den finanziellen Aspekt, sondern musste auch einen sozialen Aufstieg oder zumindest die Verhinderung eines möglichen Abstiegs bedeuten. Diesen Befund bestätigt Scholl, wenn er schlussfolgert, dass bereits im 19. Jahrhundert die Nachkommen gehobener sozialer Schichten das Ingenieurwesen „bereitwillig als eine auch für ihre soziale Herkunft akzeptable Beschäftigung ansahen. ${ }^{\prime 100}$

Eine Abgrenzung des Ingenieurberufs erfolgte nicht nur nach außen, sondern wurde von einer internen Differenzierung der Profession begleitet. Der 1909 gegründete Verein Deutscher Diplom-Ingenieure (VDDI), dessen Name bereits deutlich macht, welches Bildungspatent für eine Mitgliedschaft Bedingung war, verlieh dieser Entwicklung sichtbar Ausdruck. Die Reichweite des Vereins blieb jedoch begrenzt. 1914 zählte man lediglich 3.50o bis 4.ooo Mitglieder, was einem Anteil von 13 Prozent an der Gesamtzahl der Diplom-Ingenieure entsprach. Im selben Jahr waren jedoch im VDI gut 6.ooo Akademiker organisiert. ${ }^{101}$ Der VDDI schloss mit seiner Politik aber auch jene Ingenieure aus, die zwar eine TH besucht, hier aber keinen Abschluss erworben hatten - immerhin rund ein Drittel aller Immatrikulierten. ${ }^{102}$

An dieser Stelle wird der Fokus auf das soziale Umfeld der Regierungsbaumeister gelegt, die sich um eine Beschäftigung in den afrikanischen Kolonien bewarben. Ein geringes Prestige und Einkommen der Eltern - hier vor allem des Vaters - könnten darauf hindeuten, dass die Betreffenden die Kolonien zum einen als eine Chance des gesellschaftlichen Aufstiegs, zum anderen aber auch als Möglichkeit des schnellen Gelderwerbs wahrnahmen. Für eine quantitative Untersuchung reicht die Anzahl der Ingenieure, über die Erkenntnisse zum Elternhaus vorliegen, nicht aus. Die folgenden Ausführungen können daher nur ein Schlaglicht auf den elterlichen Hintergrund der akademisch gebildeten Ingenieure, die in der Zeit zwischen 1900 und dem Ausbruch des Ersten Weltkrieges in DSWA tätig gewesen waren, werfen. Hierbei handelte

\footnotetext{
99 Vgl. Krais, Einleitung, 1981, S. 8 f.

100 Scholl, Ingenieure in der Frühindustrialisierung, 1978, S. 219, 420; vgl. auch Fraunholz/ Wölfel, Hochmoderne Ingenieure, 2012, S. 23.

101 Gispen, Interessenkonflikte, 1994, S. 325 f. Vgl. vertiefend zum VDDI: Dietz, Technik und Kultur, 1996, v. a. S. 109.

102 Sander, Ingenieurwesen, 2008, S. 24. Auch für Techniker ohne abgeschlossene Ausbildung finden sich in den Reihen der Kolonialingenieure interessante Belege, die im Hauptteil aufgegriffen werden.
} 
es sich um jene Regierungsbaumeister, die im Staatsdienst beschäftigt waren und deren Personalakten im Bundesarchiv einsehbar sind. Ergänzt wird diese Übersicht durch einzelne Ingenieure in leitenden Positionen aus DSWA und DOA, deren Herkunftsverhältnisse aus anderen Quellen ersichtlich werden.

Von den 19 Personen, für die Personalakten des ehemaligen Gouvernements DSWA vorliegen, lassen sich für sechs Ingenieure keine Angaben finden, für die gleiche Anzahl ist in Bezug auf die (Schwieger-)Eltern der Status „Rentier“ nachzuweisen. Von den übrigen sieben Ingenieuren bekleidete lediglich der Vater Ernst Wellmanns als Geheimer Baurat ein gehobenes technisches Amt. Bei den in Ostafrika tätigen Ingenieuren sind dem Autor immerhin zwei Personen, Clemens Gillman und Manfred Heckel, bekannt, auf die dies ebenfalls zutraf. Für die übrigen akademischen Kolonialingenieure aus DSWA lassen sich an väterlichen Berufen ein Präsident des Oberlandeskulturgerichts, ein in DSWA ansässiger Pfarrer, ein Landwirt, ein Gutsbesitzer, ein Kaminkehrermeister, ein Kammermusiker an der Staatsoper Berlin sowie ein Polizeisekretär finden. ${ }^{103}$ Wie aus den Memoiren des Rolex-Gründers Hans Wilsdorf, dem Bruder des Regierungsbaumeisters und Kolonialingenieurs Karl Wilsdorf, hervorgeht, wuchsen die beiden Brüder und ihre Schwester in einem gutbürgerlichen Elternhaus auf, das sie nach deren Tod in den 189oer-Jahren mit einem umfassenden Erbe ausstattete. ${ }^{104}$ Darüber hinaus entstammte Franz Baltzer, der langjährige Vortragende Rat im RKA, einem akademischen Elternhaus. Sein Vater lehrte als Professor für Mathematik in Gießen und war zuvor bereits als Mitglied in die Sächsische Akademie der Wissenschaften in Leipzig aufgenommen worden. ${ }^{105}$ Franz Allmaras entstammte einer Pfälzer Winzerfamilie aus Alsterweil. Um Allmaras ein Studium an der Polytechnischen Hochschule in München zu ermöglichen, nahm die Mutter wohl ein Darlehen bei Verwandten auf, nachdem ihr Mann verstorben war. ${ }^{106}$

Diese Aussagen über die Herkunftsverhältnisse der Regierungsbaumeister sind zwar nur bedingt zu verallgemeinern, sie geben aber durchaus einen anschaulichen Eindruck über die in DSWA beschäftigten Ingenieure in Leitungspositionen. Diese Erkenntnisse unterstützen die Annahme, dass akademisch gebildete Ingenieure, zumindest wenn sie im Verlauf ihrer Karriere in den Kolonien arbeiteten, häufig gutbürgerlichen Verhältnissen entstammten. Zumindest die Gruppe der Hochschulingenieure stellte sich damit

103 Personalakten-DSWA, BArchB, R 1002.

104 Rolex Watch, Rolex Jubilee, 1946, S. 7 f.

105 F.: Franz Baltzer (Nachruf), 1927, S. 545 f.

106 Verband Alter Münchener Germanen, Gedenkblätter, 2013, S. 20 f. 
als deutlich bürgerlicher und weniger heterogen dar, als dies für die gesamte technische Profession gelten konnte.

\subsection{Hierarchie des Feldes}

Das deutsche technische Schulwesen zu Beginn des 20. Jahrhunderts basierte in groben Zügen auf drei Säulen, mit den Technischen Hochschulen an der Spitze, dem niedrigeren und höheren Maschinenbauwesen als mittlerer Ebene sowie einem Sockel aus Technikern, die die Möglichkeit hatten, ihr vor allem aus praktischer Arbeit und Lehre hervorgegangenes Können und Wissen berufsbegleitend zu erweitern. ${ }^{107}$ Dies entspricht der Gliederung, wie sie der Rektor des Polytechnikums in Köthen, Karl Foehr, im ersten Jahrzehnt des 2o. Jahrhunderts darstellte.

Tabelle 1 Gliederung der technischen Ausbildungswege nach Foehr, 1909

\begin{tabular}{l|l|l|l}
\hline & Empirische Berufe: & $\begin{array}{l}\text { Disponierende } \\
\text { Berufe: }\end{array}$ & Gelehrte Berufe: \\
\hline Unterstufe 1 & Volksschule & Elementarschule & Elementarschule \\
\hline Mittelstufe 2 & Bürgerschule & Realschule & Realgymnasium \\
\hline Oberstufe 3 & $\begin{array}{l}\text { Maschinenbau- } \\
\text { schule, Technikum }\end{array}$ & Polytechnikum & Technische Hochschule \\
\hline Abschluss & Techniker & Ingenieur & $\begin{array}{l}\text { Oberingenieur, } \\
\text { Doktor-Ingenieur } \\
\text { (Voraussetzung für } \\
\text { Regierungsbauführer } \\
\text { und -meister) }\end{array}$ \\
\hline
\end{tabular}

Betrachtet man nun die Vermittlung von Bildung mit Bourdieu als eine Reproduktionsstrategie bestimmter Klassen und Fraktionen, so erscheint es wenig nachvollziehbar, dass Eltern, die der bürgerlichen Schicht zuzurechnen sind, ihren Nachkommen die Finanzierung eines technischen Studiums ermöglichten, das selbigen einen sozialen Abstieg aufzwang oder ihn

107 Vgl. Kocka, Die Angestellten, 1981, S. 102. 
zumindest wahrscheinlich machte. ${ }^{108}$ Nehmen wir den erfolgreichen Besuch einer TH und das Ablegen der ersten beziehungsweise zweiten Staatsprüfung als Methode der Reproduktion der eigenen Klassenzugehörigkeit an, so lässt sich darin eher eine Strategie erkennen, „mittels dere[r] Individuen in einer sich wandelnden Gesellschaft ihre Position in der Sozialstruktur zu behaupten oder zu verbessern suchen und mittels dere[r] sie zugleich die bestehende Sozialstruktur reproduzieren. ${ }^{\text {109 }}$ Dieses Phänomen wurde bereits am Beispiel der schulischen Zugangsberechtigung für die Polytechnika und Technischen Hochschulen nachgewiesen, die sich nicht aus den praktischen Bedürfnissen der beruflichen Tätigkeit ableiteten, sondern primär einen symbolischen Wert besaßen. ${ }^{110}$ Dies trug ebenso wie die Mathematisierung des technischen Studiums zu einem Prestigegewinn der Profession bei. ${ }^{111}$

Ich vertrete aufgrund der angeführten Literatur und Quellenverweise die These, dass die Akademisierung der technischen Ausbildung mit dem Eintritt von Nachkommen aus bürgerlichen Schichten verknüpft war. Höhere Anforderungen an eine nicht technische Allgemeinbildung sowie höhere finanzielle Aufwendungen sorgten dafür, dass weniger Kindervon Handwerkern oder Arbeitern an höheren technischen Bildungsinstituten ein Studium beginnen konnten. Der Befund, dass die zunehmende Theoretisierung und Formalisierung der höheren technischen Bildung nicht auf den industriellen Bedarf abzielten, stützt die Annahme. ${ }^{112}$ Wolfgang König schreibt diesbezüglich sogar von einer nicht zu unterschätzenden Skepsis, mit der Industrieunternehmen seit den 188oer-Jahren die zunehmend an Hoch- und Mittelschulen ausgebildeten Techniker beschäftigten. Unternehmensvertreter befürchteten, dass die Zunahme theoretischer Bildung zulasten der praktischen Fähigkeiten erfolgen würde.

Aus Sicht der Industrieunternehmen orientierte sich die Akademisierung damit nicht an den fachlichen Bedürfnissen der Arbeitspraxis. Vielmehr wurde dies als problematische Entwicklung wahrgenommen, zumal die Hochschulabsolventen ähnliche Positionen in der Industrie besetzten wie die Abgänger

108 Dem berechtigten Einwand, die betreffenden Eltern hätten ihren Söhnen das bevorzugte Studium ohne Rentabilitätsgedanken finanziert, ist zu entgegnen, dass bereits der vielfache Wunsch der jungen Erwachsenen nach einem technischen Studium dessen vermeintliche allgemeine soziale Geringschätzung zweifelhaft erscheinen lässt.

109 Krais, Einleitung, 1981, S. 7; vgl. Bourdieu u. a., Kapital und Bildungskapital, 1981, S. 23-25, 81.

110 Vgl. Bourdieu, Die feinen Unterschiede, 2008, S. 456; Bourdieu/Boltanski, Titel und Stelle, 1981, S. 91-93.

111 Vgl. u. a. Neugebauer, Bildungswesen in Preußen, 1992, S. 7 O5 f.; Manegold, Emanzipation der Technik, 1977, S. 47-49.

112 Vgl. auch Manegold, Emanzipation der Technik, 1977, S. 36; König, Verein Deutscher Ingenieure, 1994, S. 3 O7. 
von Mittel- und Fachschulen. ${ }^{113}$ Dass ein staatlich garantierter schulischer Abschluss für die Ingenieure dennoch zunehmend zur Regel wurde, beweist nach Bourdieu die Unabhängigkeit der Systeme Wirtschaft und Bildung voneinander. ${ }^{114}$ Überträgt man diese Entwicklung auf das Feld der Technik, so verfügte auch dieses über eine gewisse Autonomie. Ökonomische Erwägungen wie eine kürzere Ausbildungsdauer für Techniker und Ingenieure wurden einem allgemeinen Bildungsideal untergeordnet. Dazu trug auch der Umstand bei, dass ein gewisser Teil der technischen Fachkräfte später im Staatsdienst und hier vor allem im Bauwesen beschäftigt werden sollte. ${ }^{115}$

Die Privatwirtschaft stellte den Titel des Oberingenieurs, der von Unternehmen eigenständig vergeben und dann auch außerhalb des Betriebs getragen wurde, dem staatlichen Bildungspatent entgegen. ${ }^{116}$ Die Möglichkeit, nach eigenem Ermessen Personen, die Arbeiten mit hohem technischen Anspruch ausführten, auszuzeichnen, ging aber auch mit dem Umstand einher, dass jener Titel lediglich durch das Unternehmen garantiert wurde. ${ }^{117} \mathrm{Er}$ ist somit als ein Versuch des Systems der Wirtschaft zu betrachten, seine Macht und seinen Einfluss auf das Bildungswesen auszuweiten. ${ }^{118}$ Die Ausrichtung auf eine theoretisch anspruchsvollere und vor allem für das Deutsche Reich vereinheitlichte technische Vorbildung kann somit für das ausgehende 19. Jahrhundert nicht als Antwort auf die neuen Ansprüche einer zunehmenden Verwissenschaftlichung der beruflichen Praxis gelten, sondern ist vielmehr aus Sicht der damit verbundenen Statuserhöhung der betreffenden Gruppe zu verstehen. ${ }^{119}$

Gerade vor diesem Hintergrund sind die Klagen der akademisch qualifizierten Ingenieure über fehlende Anerkennung beziehungsweise eine ihren Bildungspatenten inadäquate Stellung und Entlohnung in der Industrie als Folge der Umstrukturierung des technischen Bildungssystems gesondert zu betrachten. ${ }^{120}$ Ist es nicht durchaus denkbar, dass dieser auf eine spezielle Gruppe innerhalb der technischen Profession beschränkte Umstand für die Gesamtheit der Ingenieure verallgemeinert wurde? Die fehlende soziale

113 König, Staatsdiener, 2006, S. 199; Sander, Ingenieurwesen, 2008, S. 221-229; Kocka, Die Angestellten, 1981, S. 109; König, Ingenieure und der VDI, 1981, S. 239.

114 Vgl. Bourdieu/Boltanski, Titel und Stelle, 1981, S. 93.

115 Zum Aufkommen dieser Praxis, vgl. Scholl, Ingenieure in der Frühindustrialisierung, 1978, S. $104 \mathrm{f}$.

116 Vgl. Bourdieu u. a., Kapital und Bildungskapital, 1981, S. 46-48.

117 Vgl. Sander, Ingenieurwesen, 2008, S. 230; König, Staatsdiener, 2006, S. 206.

118 Vgl. Bourdieu/Boltanski, Titel und Stelle, 1981, S. 94-96.

119 Vgl. Kurrer, Lebenserinnerungen (1947), 2004, S. 111 f.; dazu auch Hänseroth, Technischer Fortschritt, 2013, S. 284 f.

120 Vgl. Kocka, Die Angestellten, 1981, S. 109. 
Anerkennung wäre dann vor allem ein Problem der Diplom-Ingenieure und Regierungsbaumeister gewesen. Die zeitgenössischen Äußerungen in der Zeitschrift des VDI greifen solch eine Differenzierung jedoch nicht auf, vielmehr wurde allgemein mehr Bildung als Lösung propagiert und damit eine Anpassung an bürgerliche Ideale vollzogen. Diese Assimilationsstrategie bestimmte die Emanzipationsbestrebungen der Ingenieure bis zur Jahrhundertwende. ${ }^{121}$ Kocka verweist diesbezüglich auf die hohe Wertschätzung eines bürgerlichen Bildungskanons sowie dessen enge Verknüpfung mit dem Konzept des sozialen Prestiges. ${ }^{122}$

Das sich mit dem, zur Voraussetzung erhobenen, erfolgreichen Besuch eines Gymnasiums weiter verfestigende Berechtigungswesen besaß eine immanent sozial-legitimatorische Komponente. ${ }^{123}$ Das Streben nach Prestige durch Ausweitung der Zugangsvoraussetzung wie auch des Lern- und Lehrstoffes an den Technischen Hochschulen blieb bis ins 20. Jahrhundert ein beliebtes Instrument im technischen Bildungswesen. ${ }^{124}$ Mit dieser Entwicklung verband sich jedoch auch eine zunehmende Kluft zwischen dem für ihre Arbeit notwendigen Fachwissen und dem Allgemeinwissen, das gesellschaftlichen Aufstieg zu versprechen schien. ${ }^{125}$ Unter Bezugnahme auf Bourdieu befreit das Bildungspatent als institutionalisiertes Kulturkapital den Inhaber eben auch von der Notwendigkeit, sich und seine eigenen Fähigkeiten beständig unter Beweis stellen zu müssen - im Gegensatz zu Autodidakten, die „nur sind, was sie tun“. ${ }^{126}$ Doch dies stellt nur einen relativ beschränkten Vorteil für Ingenieure und Techniker dar, eine bessere soziale und berufliche Stellung zu erreichen, wie auch ein gesteigertes Einkommen zu generieren. ${ }^{127}$

Der Regierungsbaumeister nahm ohne Frage eine besondere Stellung innerhalb der technischen Profession ein. In den Kolonien oblag ihm die technische Leitung und Planung größerer Infrastrukturprojekte, womit er an der Spitze des entsprechenden Subfeldes stand. In Preußen mussten die Anwärter auf den Titel eines Regierungsbaumeisters neben dem erfolgreichen Abschluss eines technischen Studiums insgesamt zwei gesonderte Prüfungen unter der Aufsicht einer Regierungsbehörde bestehen. Nach dem ersten Examen, das dem Studium an einer TH folgte, trugen sie die Bezeichnung Regierungsbauführer.

\footnotetext{
121 Vgl. Paulitz, Mann und Maschine, 2012, S. 4O, 120 f., 180 f.

122 Kocka, Die Angestellten, 1981, S. 92 f.

123 Vgl. ebda., S. 92.

124 Vgl. Manegold, Der VDI, 1981, S. 141.

125 Vgl. ebda., S. 142.

126 Bourdieu, Die feinen Unterschiede, 2008, S. 48; Bourdieu, Ökonomisches Kapital (Mechanismen der Macht), 1992, S. $61 \mathrm{f}$.
}

127 Vgl. Sander, Ingenieurwesen, 2008, S. 23 o. 
Im ersten Jahrzehnt des 20. Jahrhunderts wurde dieser Titel dann durch den Abschluss des Diplom-Ingenieurs ersetzt. Letzterer wurde bereits am Ende des 19. Jahrhunderts von den Hochschulen verliehen, in Preußen jedoch erst 1899 als Titel gesetzlich geschützt. ${ }^{128}$ Regierungsbaumeister wurden die Anwärter erst, nachdem sie eine zweite Staatsprüfung erfolgreich bestanden hatten. Ein Ratgeber für den Elektrotechniker schätzte das Alter der frisch gebackenen Regierungsbaumeister auf 28 Jahre, wobei die Anwärter erst nach der staatlichen Prüfung zum Regierungsbauführer, mit 25 Jahren, einer bezahlten praktischen Tätigkeit nachgehen konnten. ${ }^{129}$ Zuvor waren sie auf Unterstützungszahlungen durch Verwandte und ihre Familie angewiesen. Der Titel des Regierungsbaumeisters stellte sich unter den gemachten Vorannahmen als eine besondere Mischform dar. Gab die abgeschlossene Diplomprüfung „nur den Nachweis einer erfolgreichen theoretischen Bildung", so musste diese durch die praktische Tätigkeit auch in den Verwaltungsgeschäften eines Bauamtes über mindestens zwei Jahre ergänzt werden. ${ }^{130}$

Dieses dem juristischen Staatsexamen entlehnte duale Prinzip der Regierungsbaumeisterprüfung war ein Ergebnis der Integration der Bauingenieure in den Staatsdienst und der damit verbundenen Aufwertung der betreffenden Ingenieursgruppe zu Amtsträgern. Eine Entwicklung, mit der die deutschen Staaten auf den seit 1850 ansteigenden Bedarf an technischen Experten für den Bahnbau reagierten. ${ }^{131}$ Dies garantierte ihnen neben einer sicheren Beamtenstellung und einem guten finanziellen Auskommen auch Pensionsansprüche. ${ }^{132}$ Vor diesem Hintergrund avancierten Baubeamte innerhalb der technischen Profession zu einer als elitär wahrgenommenen und mit einem prestigeträchtigen Status ausgestatteten Gruppe. Zudem bemühten sie sich aktiv um eine Abgrenzung gegenüber anderen Akteuren im Feld der Technik. Eckhard Bolenz spricht sogar von einer Vorbildfunktion, die sie beispielsweise für die Maschineningenieure und andere Expertengruppen bis ins erste Jahrzehnt des 20. Jahrhunderts hinein einnahmen. ${ }^{33}$

Ungeachtet des Vorbildcharakters der Baubeamten sah sich auch diese Gruppe hierarchischer Benachteiligung ausgesetzt. Diese zeigte sich vor allem bei den Regierungsbaumeistern in Preußen, die nach einer bestimmten Zahl von Dienstjahren zu Regierungs- und Bauräten ernannt wurden. In den

\footnotetext{
128 Sander, Ingenieurwesen, 2008, S. 224.

129 Anonymus, Elektrotechniker, 1897, S. 10 f.

130 Anonymus, Regierungsbaumeisterprüfung, 1945.

131 Vgl. Kurrer, Lebenserinnerungen (1947), 2004, S. 112; Scholl, Ingenieure in der Frühindustrialisierung, 1978, S. 17; Bolenz, Baubeamte, 1994, S. 118-120.

132 Ebda., S. 124.

133 Ebda., S. 117; Bolenz, Technischen Berufe, 1993, S. 46.
} 
betreffenden Regierungsstellen standen den technischen vor allem solche Beamte mit juristischer Ausbildung gegenüber. Zwar waren ersteren bereits mit der Instruktion von 1817 die gleichen Rechte zugesprochen worden, dennoch besaßen die Bauräte bei sogenannten allgemeinen Entscheidungen der Regierung lediglich eine beratende Stimme. Ein Umstand, der von der technischen Intelligenz als diskriminierend und benachteiligend aufgefasst werden musste. ${ }^{134}$ Diese Auseinandersetzung zwischen Technikern und Juristen, die als "Juristenprivileg" in der staatlichen Verwaltung bereits Thema diverser Forschungsarbeiten gewesen ist, zielte vor allem auf die Frage gleicher Kompetenzen $\mathrm{ab}$, da die Regierungsräte einheitlich vergütet wurden. ${ }^{135}$ Dennoch bemängelte der Verein Deutscher Maschineningenieure noch 1881, dass der Anteil der Juristen reziprok zur Zahl der Ingenieure mit Höhe der Hierarchiestufe in der Verwaltung zunehme. ${ }^{136}$

Im Laufe des 19. Jahrhunderts mussten die im Staatsdienst tätigen Baubeamten vermehrt Aufgaben und Kontrollfunktionen übernehmen, die ihre genuine Expertise überstiegen, womit das gegen die Juristen gerichtete Argument des fehlenden technischen Sachverstandes mit wachsender technischer Ausdifferenzierung auf die Baubeamten selbst zurückfiel. ${ }^{137}$ Bereits in den 186oer-Jahren forderte daher der VDI das Ende der Bevorzugung der Baubeamten und ihre Gleichstellung mit den Maschineningenieuren, die laut Vereinszeitschrift für bestimmte staatliche Aufgaben kompetenter seien als ihre Baukollegen. ${ }^{138}$ Selbige sahen sich im Staatswesen benachteiligt, da ihnen die höheren Positionen nur bedingt offenstanden und es für sie im Bereich der Beamtenschaft damit fast keine Aufstiegschancen gab. ${ }^{139}$ 1876, Jahre bevor das Reich sein koloniales Imperium aufbauen konnte, gelang es den preußischen Maschineningenieuren, in einer neuen Prüfungsordnung für technische Studiengänge drei unterschiedliche Bereiche für den technischen Staatsdienst in Preußen festzuschreiben: das Hochbau-, Bauingenieur- und das Maschinenwesen. Damit ging auch eine zunehmende Trennung zwischen Architekten und Bauingenieuren einher. ${ }^{140}$ Im Zuge dieser Entwicklung konnten nun auch Maschineningenieure das Staatsexamen ablegen und erhielten damit die

\footnotetext{
134 Bolenz, Baubeamte, 1994, S. $121 \mathrm{f}$.

135 Weber, Ingenieure im öffentlichen Dienst, 1978, S. 6; Bolenz, Baubeamte, 1994, S. 122.

${ }_{13} 6$ König, Ingenieure in der staatlichen Verwaltung, 1994, S. 143; Weber, Ingenieure im öffentlichen Dienst, 1978, S. 8.

137 Bolenz, Baubeamte, 1994, S. 122 f.

138 Ebda., S. 126, 128; König, Ingenieure in der staatlichen Verwaltung, 1994, S. 143; Gispen, Ingenieurelite, 1994, S. 226; vgl. dazu auch Sander, Ingenieurwesen, 2008, S. 223.

139 Scholl, Ingenieur in Ausbildung, 1981, S. 6o.

140 Bolenz, Baubeamte, 1994, S. 128.
} 
Möglichkeit, als technische Beamte in die Verwaltung einzutreten. ${ }^{141}$ Gispens Schlussfolgerung, dass im 19. Jahrhundert eine institutionalisierte technische Aus- bis hin zur Hochschulbildung im Zusammenspiel mit einem durch staatliche Prüfungen garantierten Berechtigungswesen immer mehr an Bedeutung gewann, ist demnach durchaus nachvollziehbar. ${ }^{142}$

Ende des 19. Jahrhunderts trafen konjunkturelle Krisen auf eine hohe Anzahl technischer Absolventen, sodass sich die Chancen auf dem Arbeitsmarkt für Ingenieure und Techniker massiv verschlechterten. ${ }^{143}$ Dementsprechend wurde von weiteren Hochschulgründungen im technischen Bereich, beispielsweise in Münster, vorerst abgesehen. ${ }^{144}$ Dieser Umstand schlug sich in den Folgejahren auch in einer abnehmenden Zahl technischer Studierender nieder, die sich zwischen 1878 und 1883 auf knapp 3.700 fast halbierte. ${ }^{145}$ Dies trug jedoch nur sehr bedingt dazu bei, die bestehenden Probleme zu entschärfen. Die sich zum Ende des 19. Jahrhunderts wieder verbessernde Situation auf dem Arbeitsmarkt für Techniker und Ingenieure reichte nicht aus, um die nun ebenfalls wieder steigenden Zahlen der TH- und technischen Mittelschulabsolventen zu absorbieren. ${ }^{146}$

Vor dem Ersten Weltkrieg gab es im Deutschen Reich zwischen 100.000 und 150.00o Ingenieure und Techniker. Jeder Sechste von ihnen konnte zwar einen Hochschulabschluss nachweisen, der überwiegende Teil hatte jedoch eine Mittelschule besucht. Ingenieure ohne weiterführenden technischen Schulabschluss bildeten die Ausnahme. ${ }^{147}$ Betrachtet man die Verdopplung der Zahl der Ingenieure bis in die 1920er-Jahre, so ist die Kritik an einer Überfüllung des Ingenieurberufs durchaus nachvollziehbar. ${ }^{148}$ Diese Entwicklung schlug sich bereits Jahre zuvor im Brief eines Kolonialingenieurs nieder. ${ }^{149}$

Eine gewisse Sockelarbeitslosigkeit bei den technischen Experten kann bis in die 193oer-Jahre konstatiert werden. Beruhend auf einer Mitgliederbefragung des Deutschen Techniker Vereins lag diese wohl bei gut zehn Prozent. ${ }^{150}$ Von

\footnotetext{
141 König, Ingenieure in der staatlichen Verwaltung, 1994, S. 143.

142 Gispen, Ingenieurelite, 1994, S. 227-229.

143 König, Ingenieure in der staatlichen Verwaltung, 1994, S. 146.

144 König, Staatsdiener, 2006, S. 203; vgl. zur Krise: Sander, Ingenieurwesen, 2008, S. 223.

145 Braun, Anschauungen von Ingenieuren, 1978, S. 221.

146 Sander, Ingenieurwesen, 2008, S. $234 \mathrm{f}$.

147 König, Staatsdiener, 2006, S. 204.

148 Vgl. ebda., S. $219 \mathrm{f}$.

149 Vgl. Gesuch Dipl.-Ing. Marung, 6.9.1912, BArchB, R 1002/1162, fol. 9: „[...] es wird daher für mich bei der jetzigen Überfüllung des technischen Berufes sehr schwierig sein, rechtzeitig eine annehmbare Stellung zu finden.“

15 O Vorstand des Bundes der technischen Angestellten und Beamten, 25 Jahre Technikergewerkschaft, 1929, S. 73 .
} 
einer "gravierenden Ingenieursarbeitslosigkeit" in dieser Zeit zu sprechen, lehnt der emeritierte Professor für Technikgeschichte König jedoch ab, wobei er durchaus einen verstärkten Konkurrenzdruck konstatiert, der sich negativ auf Aufstiegs- und Verdienstmöglichkeiten auswirkte. ${ }^{151}$ Gleichsam ist jener Zustrom in den technischen Berufsstand ein Indiz dafür, dass zumindest in den ersten Jahrzehnten des 20. Jahrhunderts Ingenieure und Techniker nicht oder nicht mehr damit rechnen mussten, aufgrund der Wahl ihrer Beschäftigung gesellschaftlich an Prestige zu verlieren.

Die eingangs aufgeworfene Frage, was die jungen Vertreter der bürgerlichen Schicht dazu antrieb, eine Ausbildung beziehungsweise ein Studium im technischen Bereich anzustreben, ob es sich dabei lediglich um eine Ausweichbewegung auf neue Sektoren handelte, um ein gesichertes Auskommen zu erlangen, wird im folgenden Abschnitt aufgegriffen. Ein dem deutschen Kaiser, Wilhelm II., über den Stand der Techniker zugeschriebenes Bonmot aufnehmend: „Wenn jemand zwei Söhne habe, so solle er zumindest einen davon Ingenieur werden lassen“, spricht der Verband der technischen Angestellten und Beamten von einer "goldene[n] Zeit" der technischen Berufsgruppe bis zum Ausbruch der sogenannten Gründerkrise infolge des Gründerkrachs Mitte der 1870er-Jahre. Diese manifestierte sich wie beschrieben in einem Übermaß technisch ausgebildeter Kräfte, die sich einem Mangel an - zudem noch schlecht bezahlten - Arbeitsplätzen gegenübersahen, auf die Akademiker wie Mittelschulabsolventen gleichsam Anspruch erhoben. ${ }^{152}$

Eine Ausnahme stellte dabei der Außendienst größerer Industrieunternehmen dar, worauf übereinstimmend Sander als auch Kocka verweisen. Für diesen speziellen Bereich legten die Unternehmen besonderen Wert auf einen akademischen Abschluss der sie vertretenden Ingenieure und Techniker, da jene für die betreffenden Kunden und Auftraggeber im Hinblick auf ihre schulische Bildung und damit meist auch ihre familiäre Herkunft als „sozial akzeptabel" erschienen. ${ }^{153}$ Neben der tatsächlichen fachlichen Expertise der Baumeister und -räte war auch ihre meist bürgerliche Sozialisation ein entscheidendes Auswahlkriterium aufgrund der geteilten habituellen Gemeinsamkeiten mit den potenziellen Kunden. Diese Politik, die Wahl möglicher Kandidaten vor allem für Verhandlungen an und im Umfeld von größeren Bauprojekten an ihrem sich im Bildungspatent äußernden sozialen Status festzumachen, findet sich auch im kolonialen Kontext wieder. Dies

\footnotetext{
$15^{1}$ König, Staatsdiener, 2006, S. 201.

152 Vgl. Vorstand des Bundes der technischen Angestellten und Beamten, 25 Jahre Technikergewerkschaft, 1929, S. 12-14; Weber, Stellung der deutschen Techniker, 1877, S. 3 .

153 Kocka, Die Angestellten, 1981, S. 98; Sander, Ingenieurwesen, S. 23 o.
} 
wurde für Ingenieure und Techniker, die eben hierüber nicht verfügten, zu einem Problem. So finden sich auch unter den höheren Angestellten der Firma Philipp Holzmann oder Lenz \& Co, vor allem unter denjenigen, die über Entscheidungs- und Verhandlungskompetenz verfügten, zum überwiegenden Teil Ingenieure mit akademischer Vorbildung. Diesen standen vor Ort seitens des Gouvernements auch Techniker ohne Universitätsabschluss gegenüber. ${ }^{154}$

\subsection{Gründe für den Kolonialdienst}

Den kolonialen Bereich des Feldes der Technik betraten Ingenieure und Techniker zum einen als Beamte im staatlichen Kolonialdienst, zum anderen als technische Angestellte einer Baufirma wie Holzmann oder Lenz \& Co. In den Kolonien lässt sich ihre Position in Relation zur indigenen Bevölkerung, gegenüber den ansässigen Europäern und Asiaten, aber auch im Hinblick auf die kolonialen Herrschaftsstrukturen vor Ort beschreiben. Als Teil des Feldes der Technik galten auch in dessen kolonialem Subfeld ähnliche Regeln. In Deutschland erworbene Bildungstitel sowie die Dauer der Beschäftigung beispielsweise im Staatsdienst als Regierungsbaumeister gaben auch in den Kolonien den Ausschlag für die eigene hierarchische Position.

Eine naheliegende wie schwer zu beantwortende Frage ist jene nach den Gründen, die vor allem Beamte dazu bewogen, sich freiwillig für den Kolonialdienst zu verpflichten. Was gab für die Regierungsbaumeister wie Diplom-Ingenieure den Ausschlag, sich bei der Kolonialabteilung und seit 1907 beim RKA um eine Beschäftigung in den Schutzgebieten zu bewerben? Diese Frage zu stellen, ist nicht neu. Sie war und ist Ausgangspunkt verschiedener Forschungsvorhaben. ${ }^{155} \mathrm{Um}$ sie zumindest für die Profession der akademischen Ingenieure zu beantworten, ist auf die Bewerberakten beim $\mathrm{RKA}$ zu verweisen. Bis auf wenige Ausnahmen sind fast alle zerstört worden, lediglich die Ausführungen des aus Hannover stammenden Diplom-Ingenieurs Ludwig Wilhelm Bartling (geb. 1885) blieben erhalten und geben erste Hinweise zur Beantwortung der Frage. ${ }^{156}$ Zwar existieren auch in den Archiven der ehemaligen Gliedstaaten des Kaiserreiches Bewerberunterlagen, doch geben diese nur Auskunft über Namen und Stellung der Person sowie formelle

154 Rukwied, Fahrt zur Anatolischen- und Bagdadbahn, 1911; Rothe/Röhrich, Bau der Bagdadbahn, 1942.

155 Vgl. Trotha, Koloniale Herrschaft, 1994, S. 97; Brockmeyer, Kolonialschwein, 2015, S. 109.

$15^{6}$ Vgl. Personalakten-RKA, BArchB, R 10o1/910o. 
Ablehnungsgründe wie gesundheitliche Probleme. Gründe oder Ursachen für eine Bewerbung sucht man in den amtlichen Schreiben vergeblich. ${ }^{157}$

Dem ersten Briefwechsel zwischen dem Diplom-Ingenieur Bartling und dem RKA ist zu entnehmen, dass er 1912 auf eine Annonce reagierte und „bereit [sei,] die ausgeschriebene Stelle in den Kolonien anzunehmen. ${ }^{\text {“58 Über }}$ seine Beweggründe erfährt man jedoch nichts, dafür aber, wie wichtig ihm eine Aufnahme in den Kolonialdienst war. Hierfür erklärt er sich sogar bereit, eine mögliche Anstellung in Deutschland abzulehnen: „Ich möchte bemerken, dass ich gern auf die Annahme einer Stelle verzichten würde, wenn ich mit einer Ausreise im Dienste des Kolonialamtes in den nächsten 4-6 Wochen rechnen könnte" - was ihm wenige Tage später in einem Telegramm auch bestätigt wurde, zusammen mit der Aufforderung, sich bis zum 24. November 1912 auf seine Tätigkeit in Kamerun vorzubereiten. ${ }^{159}$

Als besonders hilfreich zur Beantwortung der aufgeworfenen Frage könnten sich die autobiografischen Berichte der beiden Regierungsbaumeister Franz Allmaras und Hermann Rukwied erweisen, äußerten sich doch beide über ihre persönlichen Erfahrungen und Erlebnisse in Afrika. Die eigentliche Bewerbung für den Dienst wird jedoch nur beiläufig erwähnt. Beide sprechen lediglich davon, sich bei der verantwortlichen Stelle in Berlin "gemeldet“ zu haben. Erklärungsbedürftig erschien dieser Schritt den Autoren demnach weder in den 3oer- noch in den 6oer-Jahren des 20. Jahrhunderts gewesen zu sein, als ihre Schriften publiziert wurden. Lediglich bei Rukwied findet sich die etwas lapidare Bemerkung, "[e]s trieb mich bald in die Ferne“, um den LeserInnen seine Bewerbung beim RKA zu erklären. ${ }^{160}$

Eine Ursache für die Suche nach einer kolonialen Anstellung ließe sich auch in privaten oder beruflichen Problemen beider Ingenieure in Deutschland vermuten. Doch auch zu dieser Annahme geben die Quellen keinen Anlass. Schiere Existenznot oder drohende Arbeitslosigkeit können gleichsam als Grund für die Aufnahme einer kolonialtechnischen Tätigkeit ausgeschlossen werden. Sowohl die beiden Regierungsbaumeister als auch der benannte Bartling verfügten zum Zeitpunkt ihrer Bewerbung über ein regelmäßiges Auskommen und eine Arbeitsstelle oder wie im Falle des Diplom-Ingenieurs zumindest über eine entsprechende Zusage.

157 Vgl. dazu u. a. Sächsisches Staatsarchiv, Hauptstaatsarchiv Dresden (SächStA-D), 10717, $5118 / 5120 / 5123$.

$15^{8}$ Bartling an RKA, 5·7.1912, BArchB, R 1001/9100, fol. 4.

159 Bartling an RKA, 24.10.1912, ebda., fol. 19; RKA an Bartling, 5.11.1912, ebda., fol. 22.

16o Rukwied, Erinnerungen, 1969, S. 9; Allmaras, 2000 km Eisenbahn, 1933, S. 34. 
Gerade für die höheren technischen Beamten ist die mögliche Arbeitslosigkeit aufgrund der staatlichen Rekrutierungspraxis gänzlich auszuschließen: „Vorzugsweise kommen solche Regierungsbaumeister in Frage, welche im heimischen Staatsdienste stehen", heißt es beispielsweise in einem amtlichen Schreiben über die Annahme von höheren Baubeamten zum Dienst in den Schutzgebieten. ${ }^{161}$ Diese wurden, wenn sie sich als physisch und psychisch geeignet erwiesen, von ihrer bisherigen Tätigkeit im Staatsdienst für die Dauer der kolonialen Dienstperiode „beurlaubt“. Bei Verlängerung der kolonialen Tätigkeit erfolgte, soweit den Akten zu entnehmen ist, umstandslos auch die weitere Verlängerung der Beurlaubung.

Stellvertretend für diese Abläufe, die sich detailliert in den Personalakten für die südwestafrikanischen Beamten sowie in den betreffenden Unterlagen der Landesarchive finden, soll das Beispiel des sächsischen Regierungsbaumeisters Oswald Reinhardt ausgeführt werden. Dieser hatte sich 1905, wahrscheinlich im Juli, in Berlin beworben und war nach erfolgreichen Vorverhandlungen im Dezember desselben Jahres versuchsweise beim RKA eingestellt worden. Sowohl für diesen Zeitraum war er von seiner vorherigen Tätigkeit beim Baubüro für die Bahnhofsbauten in Leipzig beurlaubt worden als auch für weitere zwei Jahre, nachdem im April 1906 seine Entsendung nach DSWA bis Ende September 1909 bestätigt worden war. ${ }^{162}$ Dem folgten zwei weitere Verlängerungen der Beurlaubung bis zum Ausbruch des Ersten Weltkrieges. ${ }^{163}$ Ging das letzte Schreiben auch mit der Bekanntgabe einher, dass Reinhardt nun die etatmäßige Stelle des Leiters für das Tiefbauwesen beim Kaiserlichen Gouvernement in DSWA innehatte, so wurde die sächsische Behörde um eine eventuelle Wiedereingliederung in den Staatsdienst gebeten, „für den Fall eintretender Tropendienstunfähigkeit bei noch vorhandener heimischer Dienstfähigkeit“. Diese Absicherung ermöglichte Regierungsbaumeistern wie Reinhardt „den Rücktritt in sein heimisches Dienstverhältnis unter Wahrung seines Dienstalters". ${ }^{164}$

Zwar waren die Heimatbehörden generell angehalten, ihren beurlaubten Beamten nach Ablauf der kolonialen Dienstperiode oder bei etwaiger Tropenuntauglichkeit die Rückkehr an ihre heimische Dienststelle zu ermöglichen, doch galt dies nur so lang, bis die Betroffenen in den Schutzgebieten

\footnotetext{
161 Annahme von höheren Baubeamten, 15.6.1907, SächStA-D, 10717, Nr. 5055, fol. 1 f.

162 Kolonialabteilung im Auswärtigen Amt (KA AA) an Sächsisches Ministerium für Auswärtige Angelegenheiten (SächMAA), 31.7.1905, ebda., Nr. 5097; KA AA an SächMAA, 4.12.1905, ebda.; KA AA an SächMAA, 30.4.1906, ebda.

163 RKA an SächMAA, 8.7.19o9, ebda.; RKA an SächMAA, 3.4.1911, ebda.

164 RKA an SächMAA, 3.4.1911, ebda.
} 
eine etatmäßige Anstellung erhielten, wobei eben auch hiervon abgewichen wurde. ${ }^{165}$ In Anbetracht dieser Praxis mussten die Regierungsbaumeister zumindest nicht um ihre erworbene Anstellung im Heimatland fürchten, wenn sie um die Versetzung in die afrikanischen Gebiete baten. Hintergrund dieser bevorzugten Behandlung war der staatliche Wunsch, genügend gut ausgebildetes technisches Fachpersonal für eine, wenn auch vorübergehende, Tätigkeit in den Schutzgebieten zu gewinnen.

Fehlende berufliche Perspektiven, wie sie vom Autor Schmitt-Imkamp dem an der TH München studierten Architekten und Regierungsbaumeister Roderich Fick zugeschrieben werden, oder gar „Verzweiflung“, die ihn bewogen habe, sich für den Kolonialdienst in Kamerun verpflichten zu lassen, scheinen eher die Ausnahme gewesen zu sein. ${ }^{166}$ Zudem widerspricht Friederike Hellerer im selben Sammelband über Roderich Fick dieser Erklärung und verweist stattdessen auf die koloniale Tätigkeit seines Vaters Adolf Eugen Gaston Fick im 19. Jahrhundert. Sie vermutet, dass sein Sohn Roderich durch „das Vorbild und die Ideenwelt seines Vaters so sehr beeinflusst [wurde], dass er 1914 Deutschland in Richtung Westafrika verließ [...].“167 Ernsthafte Zweifel sind dementsprechend an der verkürzten Erklärung angebracht, Fick sei aus schierer Not in die Kolonien gegangen. Das Argument der Alternativlosigkeit als Begründung für einen kolonialen Abzweig von der heimischen Beamtenlaufbahn findet sich jedoch wiederholt in der Forschungsliteratur. ${ }^{168}$

Grundlegend erscheint es fraglich, für sämtliche Berufs- und Beamtengruppen die gleichen Sichtweisen, Hoffnungen, Nachteile und Bewertungen im Hinblick auf das koloniale Feld und eine mögliche Karriere darin zu unterstellen. In diesem Sinne sind die häufig zitierten Aussagen des Reichskanzlers Graf Leo von Caprivi oder auch des Vaters von Heinrich Schnee, dem letzten Gouverneur DOA, zu hinterfragen, der bestürzt auf die Absicht seines Sohns reagierte, in den Kolonialdienst einzutreten. ${ }^{169}$ Selbiges ist aus Schnees Nachlass über einen Heimatbesuch zu entnehmen, bei dem er seine Familie über seinen Entschluss in Kenntnis setzte:

165 Bischoff, Kolonialbeamte, 1911, S. 12.

166 Schmitt-Imkamp, Roderich Fick, 2014, S. 19; Orth, Zürichsee, 2007, S. 21 f.

${ }_{167}$ Hellerer, Architekt Roderich Fick, 2007, S. 58 f.

168 Vgl. Brockmeyer, Kolonialschwein, 2015, S. 115 f. Die Schlussfolgerung, die Brockmeyer aus dem Nachlass des Juristen Rudolf Asimis zieht, erscheint zumindest fraglich. Einerseits betont sie seine „koloniale“ Ausrichtung bei der Wahl seiner Arbeitsthemen und der Gründung eines Vereins zur Verbreitung kolonialen Gedankenguts, um andererseits seine nach dem Studium erfolgte Entscheidung für den Kolonialdienst als Notlösung zu präsentieren.

169 Vgl. u. a. Trotha, Koloniale Herrschaft, 1994, S. 97; Brockmeyer, Kolonialschwein, 2015, S. 108; Gann/Duignan, Rulers, 1977, S. 57, 94. 
Mein guter Vater schüttelte den Kopf und meinte, ich habe das doch nicht nötig, in die Kolonien herauszugehen. Auch er hatte wie damals viele, die Auffassung, dass die Kolonien ein Betätigungsfeld für verkrachte Existenzen oder für solche sein, die irgend etwas in der Heimat gesündigt hätten. ${ }^{170}$

Hinweise auf eine Ablehnung oder Geringschätzung der Arbeit in den Kolonien sind jedoch nur bedingt zu verallgemeinern und auf die Situation der Techniker und Ingenieure zu übertragen. Zwar berichtet auch Franz Allmaras von einer ähnlichen Begebenheit, als er dem heimischen Stammtisch mitteilte, nach Afrika zu gehen, doch suchte er hieran vor allem die eigene Entschlussbereitschaft zu verdeutlichen, denn er ließ sich, ungeachtet der allgemeinen Empörung, nicht von seinem Plan abbringen. ${ }^{171}$

Die Theorie der kolonialen Situation von Gann und Duignan und deren Auswirkung auf den Kreis von Freiwilligen, die sich zum Kolonialdienst meldeten, wird auch in der gegenwärtigen Forschung noch gern zitiert. Die beiden Autoren heben dabei auf das fehlende Prestige und die geringe ökonomische Relevanz der deutschen Schutzgebiete ab, was zu einer unklaren Gemengelage im Hinblick auf die unterschiedlichen Intentionen führte. Betonen die Autoren gerade die Heterogenität der Bewerber, so bleibt in ihren Ausführungen der Eindruck bestimmend, es handle sich hierbei um ein Sammelbecken von Taugenichtsen. ${ }^{172}$ Davon abgesehen wird häufig die Intention von Juristen oder auch Offizieren untersucht, meist ist es die Reaktion des jeweiligen sozialen Umfeldes, das thematisiert und hervorgehoben wird.

Diese Fokussierung darf nicht verwundern, bedenkt man, dass in der „Kolonie der Offiziere“ - in Togo - bis 1904 knapp die Hälfte der höheren Beamtenschaft sowie in den Jahren danach gut ein Drittel dem Offiziersstand angehörte. ${ }^{173}$ Die Attraktivität des Kolonialdienstes lag für Militärs in der Möglichkeit und Wahrscheinlichkeit, im Kampf und durch kriegerische Auseinandersetzungen Prestige zu gewinnen und somit den eigenen Aufstieg in der militärischen Hierarchie des Kaiserreiches zu beschleunigen. Dazu gehörte auch die Aufwertung kolonialer Polizeiaktionen zu Feldzügen. ${ }^{174}$ Allein für Ostafrika weist Wolfgang Petter zwischen 1891 und 1905 mehr als 70 Gefechte nach, die als Kriegsdienst anerkannt wurden. Dennoch bedingte die hohe Fluktuation in den Reihen des Militärs, dass ein Korpsgeist oder eine

\footnotetext{
170 Aufzeichnungen Schnee: Über die Zeit 1896-98, o. Dat., Nr. 23, GStA PK, VI. HA NL Schnee.

171 Allmaras, $2000 \mathrm{~km}$ Eisenbahn, 1933, S. 34.

172 Gann/Duignan, Rulers, 1977, S. 56 f.

173 Trotha, Koloniale Herrschaft, 1994, S. 98 f., 102.

174 Ebda., S. 100; Gann/Duignan, Rulers, 1977, S. 63.
} 
eigenständige koloniale Militärlaufbahn nicht entstand. ${ }^{175}$ Diese Möglichkeiten des Aufstiegs verbunden mit einem eigenen Kommando lassen es demnach durchaus nachvollziehbar erscheinen, in den Kolonialdienst einzutreten, unabhängig von dessen Wahrnehmung in der breiten Öffentlichkeit. ${ }^{176}$

Daneben sind Petters Aussagen jedoch widersprüchlich, wenn er die Offiziere und ihre Gründe für den Eintritt in den Kolonialdienst beschreibt. Lassen die eingangs von ihm ausgeführten Aspekte solch einen Schritt als individuell rational und nachvollziehbar erscheinen, um in der heimischen Hierarchie aufzusteigen, kommt er dennoch zum Ergebnis: „Für ehrengerichtlich behandelte oder gesellschaftlich unmögliche Offiziere galten die Kolonien als angemessener Aufenthalt." ${ }^{177}$ Dieser Schlussfolgerung widerspricht Zurstrassen, die betont, dass der koloniale Beamtendienst im Allgemeinen keine Notlösung war oder lediglich als Zufluchtsort für gescheiterte Existenzen diente. Gerade für bürgerliche Beamte, die sich „aufstiegs- und chancenorientiert" gaben, war er eine reale Wahlmöglichkeit. Sie spricht gar von einer „Instrumentalisierung der Karriereambitionen“, um den Kolonialbeamtenstand aufzuwerten. ${ }^{178}$ Dieser Interpretation ist auch mit Blick auf die kolonialen Regierungsbaumeister zu folgen.

Ein Grund für die Bewerbung der Baumeister mögen dementsprechend die besseren Aufstiegschancen innerhalb der kolonialen Hierarchie gewesen sein, die auch das bereits zuvor zitierte Heft für Kolonialbeamte benennt. ${ }^{179}$ Doch selbst ohne eine möglicherweise höhere Stelle in den Kolonien als Eisenbahnkommissar oder Referent des Gouverneurs dürfte das durch die koloniale Zulage beträchtlich erhöhte Gehalt, ungeachtet der ebenfalls höheren Lebenshaltungskosten, Ingenieure und Techniker zum Fortgehen bewogen haben. ${ }^{180}$ Selbst die relative Bedeutungslosigkeit der Kolonien, wie sie von Gann und Duignan angeführt wurde und aus dem die beiden Autoren auf die vermeintliche Prestigelosigkeit des Kolonialdienstes schließen, mag unter umgekehrten Vorzeichen für die technischen Experten seine Berechtigung haben. Bedeutete doch für diese das Fehlen technischer Infrastruktur die Chance, genau jenem Missstand abzuhelfen. Titel biografischer Erlebnisberichte wie Ich baue $2000 \mathrm{~km}$ Eisenbahn des Regierungsbaumeisters und langjährigen

\footnotetext{
175 Petter, Offizierskorps, 1980, S. 164-166.

176 Gann/Duignan, Rulers, 1977, S. 64.

177 Petter, Offizierskorps, 1980, S. 167.

178 Zurstrassen, Stück deutscher Erde, 2008, S. 253.

179 Bischoff, Kolonialbeamte, 1911, S. 11.

180 Vgl. dazu das Kapitel 3.4 Koloniales Einkommen.
} 
Eisenbahnkommissars Allmaras deuten auf das Prestige einer kolonialen Karriere hin, wie es zumindest die leitenden Ingenieure antizipierten. ${ }^{181}$

Neben karrieristischen Motiven dürfte bei den Kolonialbeamten auch die Abenteuerlust und Autonomie eine entscheidende Rolle gespielt haben. Alfred Gottwaldt urteilt beispielsweise über Julius Dorpmüller, den späteren Reichsverkehrsminister: „für tatkräftige junge Ingenieure bot die Preußische Staatseisenbahnverwaltung nur wenige Entfaltungsmöglichkeiten“. Dies stand im krassen Kontrast zu einer leitenden Stellung in China, wie sie Dorpmüller vor dem Ersten Weltkrieg einnahm. ${ }^{182}$ Zudem bot der Kolonialdienst die Möglichkeit, Gebiete und Länder kennenzulernen, was sonst nur unter großem finanziellen Aufwand möglich gewesen wäre. Auch lässt sich für die Ingenieure in ihrem neuen Arbeitsgebiet eine gewisse Autonomie und damit die Chance nachweisen, selbstständig tätig zu sein, wie Vacher es beispielsweise für die französischen Kolonialingenieure nachweist. ${ }^{183}$ In diesem Zusammenhang ist auf die Wahrnehmung Afrikas als noch nicht technisierten, unerschlossenen Raum zu verweisen. Dementsprechend unterstelle ich den Ingenieuren und Technikern eine Ideologie der Erschließung, die die Errichtung von Infrastruktur in den Kolonien zu einem Selbstzweck aufwertete.

181 Vgl. Allmaras, $2000 \mathrm{~km}$ Eisenbahn, 1933.

182 Gottwaldt, Reichsverkehrsminister, 2004, S. 145. Zu ähnlichen Schlüssen kommt auch Peters für die Angehörigen des Militärs: Petter, Offizierskorps, 1980, S. 165.

183 Vacher, Association des Ingénieurs, 2017, S. 124; Rukwied, Erinnerungen, 1969, S. 17. 